\title{
A Combination of the Conjectures of Mordell-Lang and André-Oort
}

\author{
Richard Pink \\ Dept. of Mathematics, ETH-Zentrum, CH-8092 Zürich, Switzerland \\ pink@math.ethz.ch
}

Summary. We propose a conjecture combining the Mordell-Lang conjecture with an important special case of the André-Oort conjecture, and explain how existing results imply evidence for it.

\section{Introduction}

We begin with some remarks on the history of related conjectures; the reader wishing to skip them may turn directly to Conjecture 1.6. Let us start (arbitrarily) with the following theorem.

Theorem 1.1 (Mordell-Weil). For any abelian variety $A$ over a number field $K$, the group of rational points $A(K)$ is finitely generated.

This was proved in 1922 by Mordell [31] for elliptic curves over $\mathbb{Q}$; the general case was established by Weil [48] in 1928. Mordell also posed the following statement as a question in the case $K=\mathbb{Q}$ :

Conjecture 1.2 (Mordell). For any irreducible smooth projective algebraic curve $Z$ of genus $\geq 2$ over a number field $K$, the set of rational points $Z(K)$ is finite.

This conjecture was proved by Faltings [16], [17] in 1983. Later another proof was found by Vojta [46], simplified by Faltings [18], and recast in almost elementary terms by Bombieri [5]. For some accounts of these developments see Hindry [21], Vojta [47], or Wüstholz [49].

The Mordell conjecture can be translated into a statement about abelian varieties, as follows. If $Z(K)$ is empty, we are done. Otherwise we can embed $Z$ into its Jacobian variety $J$, such that $Z(K)=J(K) \cap Z$. By the Mordell-Weil theorem $J(K)$ is a finitely generated group. Thus with some generalization we must prove that for any abelian variety $A$ over a field of characteristic zero, any finitely generated subgroup $\Lambda \subset A$, and any irreducible curve $Z \subset A$ of genus 
$\geq 2$, the intersection $Z \cap \Lambda$ is finite. Since this can be verified over the base field $\mathbb{C}$, the Mordell conjecture has thus been translated into a statement about subvarieties of complex abelian varieties, involving only complex algebraic and/or analytic geometry.

However, this point of view does not really help in proving the Mordell conjecture. It merely hides the inherent arithmetic nature of the problem that is introduced by the subgroup $\Lambda$. In fact, the only known proof of the statement consists in deducing it from the Mordell conjecture.

Nevertheless, the idea to study intersections like $Z \cap \Lambda$ led to other fruitful developments. Motivated by their attempts to prove the Mordell conjecture, Manin and Mumford independently raised the following statement as a question.

Conjecture 1.3 (Manin-Mumford). Let $A$ be an abelian variety over $\mathbb{C}$ and let $A_{\text {tor }}$ denote its subgroup of all torsion points. Let $Z \subset A$ be an irreducible closed algebraic subvariety such that $Z \cap A_{\text {tor }}$ is Zariski dense in $Z$. Then $Z$ is a translate of an abelian subvariety of $A$ by a torsion point.

This conjecture has been proved in several remarkably different ways. After a partial result by Bogomolov [4], the first full proof was published in 1983 by Raynaud [36], [37]. A different proof for curves was given by Coleman [8]. In the meantime other full proofs were found by Hindry [20], by Hrushovski [23], by Ullmo [44] and Zhang [52], and by Pink-Roessler [34], see also Roessler [39]. Ullmo and Zhang actually prove a stronger statement conjectured by Bogomolov, where the torsion points are replaced by all points of sufficiently small Néron-Tate height.

Lang [24], [25] combined the preceding conjectures into a single one by starting with a finitely generated subgroup $\Lambda_{0} \subset A$ and considering its division group

$$
\Lambda:=\left\{a \in A \mid \exists n \in \mathbb{Z}^{>0}: n a \in \Lambda_{0}\right\},
$$

which contains both $\Lambda_{0}$ and $A_{\text {tor }}$.

Conjecture 1.4 (Mordell-Lang). Let $A$ be an abelian variety over $\mathbb{C}$ and $\Lambda \subset A$ as above. Let $Z \subset A$ be an irreducible closed algebraic subvariety such that $Z \cap \Lambda$ is Zariski dense in $Z$. Then $Z$ is a translate of an abelian subvariety of $A$.

This conjecture, too, is now a theorem by the combination of work of Faltings [18], [19], Raynaud [35], Vojta [46], and Hindry [21]. Detailed surveys may be found in [12], [49], [47]; for a short historical overview see [22, pp.435439]. Moreover, McQuillan [26] established the natural generalization to semiabelian varieties.

Let us now turn to Shimura varieties. (The relevant notions and notation will be reviewed in Sections 2 through 4.) The prototype for all Shimura varieties is the Siegel moduli space of principally polarized abelian varieties of 
dimension $g$ with a level structure. The points in this moduli space corresponding to abelian varieties with complex multiplication are called special points, and they play a particularly important role. A major reason for this is the Shimura-Taniyama theorem [43], [9, Th. 4.19], which describes the action of the Galois group on the torsion points of any CM abelian variety. This theorem, and its generalization by Milne-Shih [11] to Galois conjugates of CM abelian varieties, determine the Galois action on special points fairly completely. The concept of special points and the results concerning the Galois action on them have also been generalized to arbitrary Shimura varieties: see Deligne [10] and Milne [27], [28].

Aside from this explicit connection between the special points on Shimura varieties and the torsion points on abelian varieties, there are several other formal analogies. For one thing, both kinds of points are those for which certain associated Galois representations have particularly small images. For another, they are invariant under an inherent additional structure, in that the torsion points on an abelian variety form a subgroup, and the set of special points on a Shimura variety is invariant under all Hecke operators. Also, both form dense subsets in the analytic topology.

This strong connection was one motivation for André [1, p.215, Problem 1] and Oort [32] around 1990 to independently pose an analogue of the ManinMumford conjecture, whose combination is nowadays phrased as follows. Oort [32] was motivated in part by a question of Coleman concerning the number of special points in the Torelli locus. There are also interesting connections with complex analytic and $p$-adic linearity properties of Shimura subvarieties: see Moonen [29, IV.1.2] and especially [30, §6].

An irreducible component of a Shimura subvariety of a Shimura variety $S$, or of its image under a Hecke operator, is called a special subvariety of $S$. Moonen, Edixhoven, and Yafaev call these 'subvarieties of Hodge type', due to their description as connected components of loci of Hodge classes.

Conjecture 1.5 (André-Oort). Let $S$ be a Shimura variety over $\mathbb{C}$, and let $\Lambda \subset S$ denote the set of all its special points. Let $Z \subset S$ be an irreducible closed algebraic subvariety such that $Z \cap \Lambda$ is Zariski dense in $Z$. Then $Z$ is a special subvariety of $S$.

This conjecture remains open, although it has been established in special cases or under additional assumptions by Moonen [29], André [2], Edixhoven [13], [14], Edixhoven-Yafaev [15], and Yafaev [51], [50]. In particular it has been proved by Edixhoven-Yafaev [15] when $Z$ is a curve and $\Lambda$ is replaced by the set of special points in a single generalized Hecke orbit.

The Manin-Mumford and André-Oort conjectures are not only related by analogy, but the obvious generalization of the latter to mixed Shimura varieties includes the former for CM abelian varieties. Namely, let $A \rightarrow S$ denote the universal family of abelian varieties over some Siegel moduli space. Then $A$ is 
a mixed Shimura variety in the sense of [33], and the special points in $A$ are precisely the torsion points in the fibers $A_{s}$ over all special points $s \in S$. On the other hand an irreducible subvariety $Z \subset A_{s}$ is an irreducible component of a mixed Shimura subvariety of $A$ if and only if $Z$ is a translate of an abelian subvariety of $A_{s}$ by a torsion point. Thus the Manin-Mumford conjecture for the CM abelian variety $A_{s}$ becomes a special case of the generalization of the André-Oort conjecture to $A$.

Building on this observation, André [3, Lect. III] suggested a 'generalized André-Oort conjecture', which for mixed Shimura varieties becomes the direct generalization of the André-Oort conjecture, but which also contains the Manin-Mumford conjecture for arbitrary abelian varieties.

The aim of this article is to generalize the correspondence just explained in another direction and to propose a conjecture about subvarieties of mixed Shimura varieties which contains not only the general Manin-Mumford conjecture, but also the general Mordell-Lang conjecture, as well as an important special case of the André-Oort conjecture. The key observation is that in the above situation, the special points in $A_{s}$ are all contained in a single Hecke orbit. Already in 1989 André [1, p.216, Problem 3] had posed a problem about subvarieties containing a dense subset of points from a Hecke orbit, and the conjecture below can be viewed as an attempt to give a precise answer to André's question. For this, the notion of special subvarieties must be generalized as follows.

Consider Shimura morphisms of mixed Shimura varieties $T^{\prime} \stackrel{[\varphi]}{\longleftarrow} \stackrel{[i]}{\longrightarrow} S$ and a point $t^{\prime} \in T^{\prime}$. An irreducible component of $[i]\left([\varphi]^{-1}\left(t^{\prime}\right)\right)$, or of its image under a Hecke operator, is called a weakly special subvariety of $S$. The proposed conjecture is this:

Conjecture 1.6. Let $S$ be a mixed Shimura variety over $\mathbb{C}$ and $\Lambda \subset S$ the generalized Hecke orbit of a point $s \in S$. Let $Z \subset S$ be an irreducible closed algebraic subvariety such that $Z \cap \Lambda$ is Zariski dense in $Z$. Then $Z$ is a weakly special subvariety of $S$.

The notions of mixed Shimura varieties, Shimura morphisms, and generalized Hecke orbits are explained in Sections 2 and 3. Special and weakly special subvarieties are studied in Section 4, and their relation with special points in Section 4. The interplay between Hodge and Galois theoretic properties of points is discussed in Section 6. On this basis we give three different kinds of evidence for the conjecture by relating it to known results:

- In Section 4 we show that, for a pure Shimura variety and the generalized Hecke orbit of a special point, the conjecture becomes a particular case of the André-Oort conjecture. If in addition $Z$ is a curve, it is thus proved by Edixhoven and Yafaev [15].

- In Section 5 we show that for subvarieties of an abelian variety which is the fiber of a Shimura morphism to a pure Shimura variety, the conjecture is equivalent to the Mordell-Lang conjecture, which is also known. 
- In Section 7 we deduce the conjecture for the Siegel moduli space and the generalized Hecke orbit of a Galois generic point from equidistribution results of Clozel, Oh, and Ullmo [6], [7].

In each case the reduction is relatively simple, in spite of the notational complexity. All the hard work is done in the cited literature. One may hope to prove the conjecture eventually by a combination of the individual approaches.

Finally, I am not sure how to generalize Conjecture 1.6 reasonably to include the full André-Oort conjecture.

The idea for this article arose in December 2003 at the workshop on "Special Points in Shimura Varieties" at the Lorentz Center in Leiden. It is my pleasure to thank the organizers for their invitation, and Y. André, D. Bertrand, L. Clozel, B. Edixhoven, B. Moonen, F. Oort, E. Ullmo, J. Wildeshaus, and A. Yafaev for interesting conversations and suggestions. I would also like to thank Y. Tschinkel for the invitation to the conference on "Diophantine Geometry" in Göttingen in June 2004, and G. Faltings, G. Harder, and N. Katz for the invitation to the conference on "Arithmetic Algebraic Geometry" in Oberwolfach in August 2004, and the opportunity to present a part of this work there.

\section{Connected mixed Shimura varieties}

In this section we review the necessary facts about mixed Shimura varieties over the complex numbers. In order to simplify the definitions and to avoid the language of adèles we restrict ourselves to connected mixed Shimura varieties, which are simply the connected components of usual mixed Shimura varieties. For the basic theory of pure Shimura varieties see Deligne [9], [10], for mixed Shimura varieties see Milne [28] or Pink [33].

First we recall some facts about mixed Hodge structures. Consider the real torus $\mathbb{S}:=\mathcal{R}_{\mathbb{C} / \mathbb{R}} \mathbb{G}_{m, \mathbb{C}}$ defined by Weil restriction. By construction it comes with a natural isomorphism $\mathbb{S}(\mathbb{R}) \cong \mathbb{C}^{*}$. For any linear representation of $\mathbb{S}$ on a complex vector space $V_{\mathbb{C}}$ and any pair of integers $(p, q)$ we let $V^{p, q}$ be the subspace of $V_{\mathbb{C}}$ on which $z \in \mathbb{S}(\mathbb{R}) \cong \mathbb{C}^{*}$ acts through multiplication by $z^{-p} \bar{z}^{-q}$. The induced $\mathbb{Z}$-filtrations

$$
F^{p} V_{\mathbb{C}}:=\bigoplus_{\substack{p^{\prime}, q \in \mathbb{Z} \\ p^{\prime} \geq p}} V^{p^{\prime}, q} \quad \text { and } \quad W_{n} V_{\mathbb{C}}:=\bigoplus_{\substack{p, q \in \mathbb{Z} \\ p+q \leq n}} V^{p, q}
$$

are called the associated Hodge and weight filtrations. If $V_{\mathbb{C}}$ is the complexification of a $\mathbb{Q}$-vector space $V$, these filtrations define a rational mixed Hodge structure on $V$ if and only if the subspace $W_{n} V_{\mathbb{C}}$ is defined over $\mathbb{Q}$ for all $n$, and $\overline{V^{p, q}} \equiv V^{q, p} \bmod W_{p+q-1} V_{\mathbb{C}}$ for all $(p, q)$. This is not the usual construction of rational mixed Hodge structures, but every rational mixed Hodge structure 
arises in this way, although the representation of $\mathbb{S}$ on $V_{\mathbb{C}}$ is not uniquely determined by the filtrations. The mixed Hodge structure is called of type $S \subset \mathbb{Z}^{2}$ if $V^{p, q}=0$ for all $(p, q) \notin S$.

Note that giving a representation of $\mathbb{S}$ on $V_{\mathbb{C}}$ is equivalent to giving a homomorphism $\mathbb{S}_{\mathbb{C}} \rightarrow \operatorname{Aut}_{\mathbb{C}}\left(V_{\mathbb{C}}\right)$ of algebraic groups over $\mathbb{C}$. In the following definition we consider more generally the set of homomorphisms $\operatorname{Hom}\left(\mathbb{S}_{\mathbb{C}}, P_{\mathbb{C}}\right)$ for a linear algebraic group $P$ over $\mathbb{Q}$. The group $P(\mathbb{C})$ acts on this set from the left by composition with the inner automorphisms int $(p): p^{\prime} \mapsto p p^{\prime} p^{-1}$ for all $p \in P(\mathbb{C})$.

Definition 2.1. A connected mixed Shimura datum is a pair $\left(P, X^{+}\right)$where

(a) $P$ is a connected linear algebraic group over $\mathbb{Q}$, with unipotent radical $W$, and with another algebraic subgroup $U \subset W$ that is normal in $P$ and uniquely determined by $X^{+}$using condition (iii) below, and

(b) $X^{+} \subset \operatorname{Hom}\left(\mathbb{S}_{\mathbb{C}}, P_{\mathbb{C}}\right)$ is a connected component of an orbit under the subgroup $P(\mathbb{R}) \cdot U(\mathbb{C}) \subset P(\mathbb{C})$,

such that for some (or equivalently for all) $x \in X^{+}$,

(i) the composite homomorphism $\mathbb{S}_{\mathbb{C}} \stackrel{x}{\rightarrow} P_{\mathbb{C}} \rightarrow(P / U)_{\mathbb{C}}$ is defined over $\mathbb{R}$,

(ii) the adjoint representation induces on Lie $P$ a rational mixed Hodge structure of type

$$
\{(-1,1),(0,0),(1,-1)\} \cup\{(-1,0),(0,-1)\} \cup\{(-1,-1)\},
$$

(iii) the weight filtration on Lie $P$ is given by

$$
W_{n}(\operatorname{Lie} P)=\left\{\begin{array}{cc}
0 & \text { if } n<-2, \\
\operatorname{Lie} U & \text { if } n=-2, \\
\operatorname{Lie} W & \text { if } n=-1, \\
\operatorname{Lie} P & \text { if } n \geq 0,
\end{array}\right.
$$

(iv) the conjugation by $x(\sqrt{-1})$ induces a Cartan involution on $(P / W)_{\mathbb{R}}^{\mathrm{ad}}$,

(v) $P / P^{\text {der }}$ is an almost direct product of a $\mathbb{Q}$-split torus with a torus of compact type defined over $\mathbb{Q}$, and

(vi) $P$ possesses no proper normal subgroup $P^{\prime}$ defined over $\mathbb{Q}$, such that $x$ factors through $P_{\mathbb{C}}^{\prime} \subset P_{\mathbb{C}}$.

If in addition $P$ is reductive, $\left(P, X^{+}\right)$is called a connected (pure) Shimura datum.

Remark 2.2. The axioms (v) and (vi) are sometimes weakened. It suffices to assume the consequence of (v) that the center of $P / W$ acts on Lie $U$ and on Lie $W / U$ through an almost direct product of a $\mathbb{Q}$-split torus with a torus of compact type defined over $\mathbb{Q}$.

Note that (v) also implies that every sufficiently small congruence subgroup of $P(\mathbb{Q})$ is contained in $P^{\text {der }}(\mathbb{Q})$. 
Facts 2.3. [33, 1.18, 3.3, 9.24] Let $\left(P, X^{+}\right)$be a connected mixed Shimura datum.

(a) The stabilizer $P(\mathbb{R})^{+}$of $X^{+} \subset \operatorname{Hom}\left(\mathbb{S}_{\mathbb{C}}, P_{\mathbb{C}}\right)$ is open in $P(\mathbb{R})$.

(b) $X^{+}$possesses a unique structure of complex manifold such that for every representation $\rho$ of $P$ on a complex vector space $V_{\mathbb{C}}$ the Hodge filtration determined by $\rho \circ x$ varies holomorphically with $x \in X^{+}$. In particular this complex structure is invariant under $P(\mathbb{R})^{+} \cdot U(\mathbb{C})$.

(c) Set $P(\mathbb{Q})^{+}:=P(\mathbb{Q}) \cap P(\mathbb{R})^{+}$. Any congruence subgroup $\Gamma \subset P(\mathbb{Q})^{+}$acts properly discontinuously on $X^{+}$, so that $\Gamma \backslash X^{+}$is a complex analytic space with at most finite quotient singularities.

(d) Every sufficiently small congruence subgroup $\Gamma \subset P(\mathbb{Q})^{+}$acts freely on $X^{+}$, so that $\Gamma \backslash X^{+}$is a complex manifold and $X^{+} \rightarrow \Gamma \backslash X^{+}$an unramified covering.

(e) $\Gamma \backslash X^{+}$possesses a natural structure of quasiprojective algebraic variety over $\mathbb{C}$.

Definition 2.4. The variety $\Gamma \backslash X^{+}$from 2.3 is called the connected mixed Shimura variety associated to $\left(P, X^{+}\right)$and $\Gamma$. It is called a connected (pure) Shimura variety if $P$ reductive. The residue class of an element $x \in X^{+}$is denoted $[x] \in \Gamma \backslash X^{+}$.

Definition 2.5. A morphism of connected mixed Shimura data $\left(P, X^{+}\right) \rightarrow$ $\left(P^{\prime}, X^{\prime+}\right)$ is a homomorphism $\varphi: P \rightarrow P^{\prime}$ of algebraic groups over $\mathbb{Q}$ which induces a map $X^{+} \rightarrow X^{\prime+}, x \mapsto \varphi \circ x$.

Facts 2.6. [33, 3.4, 9.24] Let $\varphi:\left(P, X^{+}\right) \rightarrow\left(P^{\prime}, X^{\prime+}\right)$ be a morphism of connected mixed Shimura data and $\Gamma \subset P(\mathbb{Q})^{+}$and $\Gamma^{\prime} \subset P^{\prime}(\mathbb{Q})^{+}$congruence subgroups such that $\varphi(\Gamma) \subset \Gamma^{\prime}$. Then the map

$$
[\varphi]: \Gamma \backslash X^{+} \rightarrow \Gamma^{\prime} \backslash X^{\prime+},[x] \mapsto[\varphi \circ x]
$$

is well-defined, holomorphic, and algebraic with respect to the algebraic structures from $2.3(e)$. Furthermore, $[\varphi]$ is

(a) a finite morphism if the identity component of $\operatorname{Ker}(\varphi)$ is a torus,

(b) surjective if $\operatorname{Im}(\varphi)$ contains the derived group $\left(P^{\prime}\right)^{\mathrm{der}}$, and

(c) a (possibly ramified) covering if the conditions in (a) and (b) both hold.

Definition 2.7. Any morphism $[\varphi]$ as in 2.6 is called a Shimura morphism. Moreover, it is called a

(a) Shimura immersion if the condition in 2.6 (a) holds,

(b) Shimura submersion if the condition in 2.6 (b) holds,

(c) Shimura covering if the condition in 2.6 (c) holds.

Here the word 'immersion' is intended to reflect its meaning in topology, where it is a purely local condition. Any composite of an unramified covering 
and a closed embedding is an immersion in this sense. The use in 2.7 (a) includes ramified coverings and is therefore somewhat abusive, but I did not find a better word.

Proposition 2.8. Any Shimura morphism $[\varphi]$ possesses a factorization into Shimura morphisms $[\varphi]=[i] \circ[\psi] \circ[\pi]$, where

(a) $\pi$ is surjective, and its kernel is connected and possesses no non-trivial torus quotient,

(b) $\psi$ is surjective and the identity component of its kernel is a torus, and

(c) $i$ is injective.

In particular, $[\pi]$ is a Shimura submersion, $[\psi]$ a Shimura covering, and $[i]$ a Shimura immersion.

Proof. Let $\varphi:\left(P, X^{+}\right) \rightarrow\left(P^{\prime}, X^{\prime+}\right)$ be any morphism of connected mixed Shimura data. Let $K \triangleleft P$ denote the identity component of $\operatorname{Ker}(\varphi)$, and let $K^{\prime}$ be the smallest connected normal subgroup of $K$ such that $K / K^{\prime}$ is a torus. This is a characteristic subgroup of $K$ and therefore normal in $P$. Set $\bar{P}:=P / K^{\prime}$ and $\bar{P}^{\prime}:=\varphi(P) \subset P^{\prime}$. Then $\varphi$ is the composite of natural homomorphisms

$$
P \stackrel{\pi}{\longrightarrow} \bar{P} \stackrel{\psi}{\longrightarrow} \bar{P}^{\prime} \stackrel{i}{\longrightarrow} P^{\prime}
$$

which possess the desired properties $(\mathrm{a}-\mathrm{c})$. Let

$$
\bar{X}^{+} \subset \operatorname{Hom}\left(\mathbb{S}_{\mathbb{C}}, \bar{P}_{\mathbb{C}}\right) \quad \text { and } \quad \bar{X}^{\prime+} \subset \operatorname{Hom}\left(\mathbb{S}_{\mathbb{C}}, \bar{P}_{\mathbb{C}}^{\prime}\right)
$$

denote the images of $X^{+} \subset \operatorname{Hom}\left(\mathbb{S}_{\mathbb{C}}, P_{\mathbb{C}}\right)$ under composition with $\pi$ and $\psi \circ \pi$, respectively. Since the homomorphisms

$$
P(\mathbb{R}) \stackrel{\pi}{\longrightarrow} \bar{P}(\mathbb{R}) \stackrel{\psi}{\longrightarrow} \bar{P}^{\prime}(\mathbb{R})
$$

induce surjections on the identity components, one can easily verify that $\left(\bar{P}, \bar{X}^{+}\right)$and $\left(\bar{P}^{\prime}, \bar{X}^{\prime+}\right)$ are connected mixed Shimura data. Thus $[\varphi]$ factors through Shimura morphisms

$$
\Gamma \backslash X^{+} \stackrel{[\pi]}{\longrightarrow} \bar{\Gamma} \backslash \bar{X}^{+} \stackrel{[\psi]}{\longrightarrow} \bar{\Gamma}^{\prime} \backslash \bar{X}^{\prime+} \stackrel{[i]}{\longrightarrow} \Gamma^{\prime} \backslash X^{\prime+}
$$

for suitable congruence subgroups, as desired.

Construction 2.9. Let $\left(P, X^{+}\right)$be a connected mixed Shimura datum and $V$ a finite dimensional representation of $P$ over $\mathbb{Q}$. Assume that for some (or equivalently for all) $x \in X^{+}$the induced rational mixed Hodge structure on $V$ has type $\{(-1,0),(0,-1)\}$. With

$$
V_{\mathrm{alg}}:=V \otimes_{\mathbb{Q}} \mathbb{G}_{a, \mathbb{Q}} \cong \mathbb{G}_{a, \mathbb{Q}}^{\operatorname{dim} V}
$$

we can define the semidirect product $P \ltimes V_{\text {alg }}$ as a linear algebraic group. Set $V_{\mathbb{R}}:=V \otimes_{\mathbb{Q}} \mathbb{R} \cong V_{\text {alg }}(\mathbb{R})$ and let 


$$
X^{+} \ltimes V_{\mathbb{R}} \subset \operatorname{Hom}\left(\mathbb{S}_{\mathbb{C}}, P_{\mathbb{C}} \ltimes V_{\mathrm{alg}, \mathbb{C}}\right)
$$

denote the conjugacy class under

$$
\left(P(\mathbb{R})^{+} \cdot U(\mathbb{C})\right) \ltimes V_{\mathbb{R}}=\left(P \ltimes V_{\text {alg }}\right)(\mathbb{R})^{+} \cdot U(\mathbb{C})
$$

generated by $X^{+} \subset \operatorname{Hom}\left(\mathbb{S}_{\mathbb{C}}, P_{\mathbb{C}}\right)$. The notation $X^{+} \ltimes V_{\mathbb{R}}$ is justified by the natural bijection

$$
X^{+} \times V_{\mathbb{R}} \stackrel{\sim}{\longrightarrow} X^{+} \ltimes V_{\mathbb{R}},\left(x, v^{\prime}\right) \mapsto \operatorname{int}\left(v^{\prime}\right) \circ x .
$$

Under this bijection the action of $(p, v) \in\left(P(\mathbb{R})^{+} \cdot U(\mathbb{C})\right) \ltimes V_{\mathbb{R}}$ corresponds to the twisted action $(p, v) \cdot\left(x, v^{\prime}\right)=\left(p x, p v^{\prime}+v\right)$. Moreover the pair $\left(P \ltimes V_{\text {alg }}, X^{+} \ltimes V_{\mathbb{R}}\right)$ is a connected mixed Shimura datum. Let $\Gamma \subset P(\mathbb{Q})^{+}$be a sufficiently small congruence subgroup and $\Gamma_{V} \subset V=V_{\text {alg }}(\mathbb{Q})$ a $\Gamma$-invariant $\mathbb{Z}$-lattice of $\operatorname{rank} \operatorname{dim}(V)$. The projection $\pi: P \ltimes V_{\text {alg }} \rightarrow P$ then induces a Shimura epimorphism

$$
A:=\left(\Gamma \ltimes \Gamma_{V}\right) \backslash\left(X^{+} \ltimes V_{\mathbb{R}}\right) \stackrel{[\pi]}{\longrightarrow} \Gamma \backslash X^{+}=: S .
$$

Furthermore the homomorphisms

$$
\begin{aligned}
& P \ltimes V_{\text {alg }}^{2} \stackrel{\mu}{\longrightarrow} P \ltimes V_{\text {alg }} \stackrel{\pi}{\longrightarrow} P \stackrel{\epsilon}{\longrightarrow} P \ltimes V_{\text {alg }}, \\
& \left(p, v, v^{\prime}\right) \longmapsto\left(p, v+v^{\prime}\right) \longmapsto \\
& \longrightarrow
\end{aligned}
$$

induce Shimura morphisms

$$
\begin{gathered}
\left(\Gamma \ltimes \Gamma_{V}^{2}\right) \backslash\left(X^{+} \ltimes V_{\mathbb{R}}^{2}\right) \longrightarrow\left(\Gamma \ltimes \Gamma_{V}\right) \backslash\left(X^{+} \ltimes V_{\mathbb{R}}\right) \longrightarrow \Gamma \backslash X^{+} \longrightarrow\left(\Gamma \ltimes \Gamma_{V}\right) \backslash\left(X^{+} \ltimes V_{\mathbb{R}}\right) \\
\qquad \| \\
A \times S A \longrightarrow
\end{gathered}
$$

which turn $A$ into a family of abelian varieties over $S$, for which $[\mu]$ is the addition morphism and $[\epsilon]$ the zero section. All its fibers are isomorphic to the compact torus $\Gamma_{V} \backslash V_{\mathbb{R}} \cong \Gamma_{V} \otimes(\mathbb{Z} \backslash \mathbb{R})$, whose complex structure depends on the base point in $S$. (Compare [33, 3.13-14].)

Example 2.12. Consider an integer $g>0$ and a non-degenerate alternating $2 g \times 2 g$-matrix $E$ over $\mathbb{Q}$. The associated group of symplectic similitudes is

$$
\mathrm{CSp}_{2 g, \mathbb{Q}}:=\left\{U \in \mathrm{GL}_{2 g, \mathbb{Q}} \mid \exists \lambda \in \mathbb{G}_{m, \mathbb{Q}}: U^{t} E U=\lambda E\right\} .
$$

Let $\mathcal{H}_{2 g}$ be the set of homomorphisms $h: \mathbb{S} \rightarrow \mathrm{CSp}_{2 g, \mathbb{R}}$ that induce a Hodge structure of type $\{(-1,0),(0,-1)\}$ on the tautological representation $\mathbb{Q}^{2 g}$, for which the symmetric pairing

$$
\mathbb{R}^{2 g} \times \mathbb{R}^{2 g} \rightarrow \mathbb{R},\left(v, v^{\prime}\right) \mapsto v^{t} E h(\sqrt{-1}) v^{\prime}
$$


is positive definite. It is known that the identity component of $\mathrm{CSp}_{2 g}(\mathbb{R})$ acts transitively on $\mathcal{H}_{2 g}$ and the pair $\left(\mathrm{CSp}_{2 g, \mathbb{Q}}, \mathcal{H}_{2 g}\right)$ is a connected pure Shimura datum. For suitable congruence subgroups the associated connected Shimura variety is a fine moduli space for certain polarized abelian varieties of dimension $g$ with a level structure.

Moreover, we can apply Construction 2.9 to the tautological representation of $\mathrm{CSp}_{2 g, \mathbb{Q}}$ on $V_{2 g}:=\mathbb{Q}^{2 g}$, yielding a connected mixed Shimura datum with underlying group $\mathrm{CSp}_{2 g, \mathbb{Q}} \ltimes \mathbb{G}_{a, \mathbb{Q}}^{2 g}$. For suitable congruence subgroups the associated connected mixed Shimura variety is the universal family of abelian varieties over the moduli space. Every abelian variety can be realized as a fiber of such a family. (See $[33,2.7, \S 10]$.

Remark 2.13. The above construction can be generalized to semi-abelian varieties. For a brief sketch let $\left(P, X^{+}\right)$and $V$ be as in Construction 2.9. Suppose that, as in Example 2.12, we are given a $P$-equivariant non-degenerate alternating form $\Psi: V \times V \rightarrow U \cong \mathbb{Q}$, where $U$ is a representation of $P$ of Hodge type $\{(-1,-1)\}$. For any positive integer $d$ let $P \ltimes V_{\text {alg }}^{d}$ act on $V \oplus U^{d}$ by the representation

$$
\left(p,\left(v_{i}\right)\right) \cdot\left(v,\left(u_{i}\right)\right):=\left(p v,\left(p u_{i}+\Psi\left(v_{i}, v\right)\right)\right) .
$$

Let $\Gamma_{V}, \Gamma_{V}^{*} \subset V$ and $\Gamma_{U} \subset U$ be $\Gamma$-invariant $\mathbb{Z}$-lattices such that $\Psi$ induces a perfect pairing $\Gamma_{V} \times \Gamma_{V}^{*} \rightarrow \Gamma_{U}$. Then the projection homomorphisms

$$
\left(P \ltimes V_{\text {alg }}^{d}\right) \ltimes\left(V \oplus U^{d}\right)_{\text {alg }} \rightarrow P \ltimes V_{\text {alg }}^{d} \rightarrow P
$$

and

$$
\left(\Gamma \ltimes \Gamma_{V}^{d}\right) \ltimes\left(\Gamma_{V}^{*} \times \Gamma_{U}^{d}\right) \rightarrow \Gamma \ltimes \Gamma_{V}^{d} \rightarrow \Gamma
$$

induce Shimura epimorphisms $B \rightarrow A^{d} \rightarrow S$. Here $B \rightarrow A^{d}$ is a family of algebraic groups, whose fibers are isomorphic to

$$
\Gamma_{V}^{*} \backslash V_{\mathbb{R}} \oplus\left(\Gamma_{U} \backslash U_{\mathbb{C}}\right)^{d}
$$

with a twisted complex structure determined by the base point in $A^{d}$. One verifies that this is a semi-abelian variety with a torus part of dimension $d$. More precisely, let $A_{s}$ be the fiber above $s \in S$ of the family $A \rightarrow S$ in Construction 2.9. Let $A_{s}^{*}$ denote the abelian variety dual to $A_{s}$. Then $A_{s}^{d} \cong$ $\operatorname{Ext}^{1}\left(A_{s}^{*}, \mathbb{G}_{m}^{d}\right)$, and the fiber of $B \rightarrow A^{d}$ above a point $a \in A_{s}^{d}$ is the associated extension

$$
1 \rightarrow \mathbb{G}_{m}^{d} \rightarrow B_{a} \rightarrow A_{s}^{*} \rightarrow 1
$$

Every semi-abelian variety is isomorphic to such a $B_{a}$ (compare [33, 3.13-14]). 


\section{Generalized Hecke orbits}

Let $S=\Gamma \backslash X^{+}$be the connected mixed Shimura variety associated to a connected mixed Shimura datum $\left(P, X^{+}\right)$and a congruence subgroup $\Gamma \subset$ $P(\mathbb{Q})^{+}$.

\section{Definition 3.1.}

(a) For any automorphism $\varphi$ of $P$ that induces an automorphism of Shimura data $\left(P, X^{+}\right) \rightarrow\left(P, X^{+}\right)$, the diagram of Shimura coverings

$$
S=\Gamma \backslash X^{+} \stackrel{[\mathrm{id}]}{\longleftarrow}\left(\Gamma \cap \varphi^{-1}(\Gamma)\right) \backslash X^{+} \stackrel{[\varphi]}{\longrightarrow} \Gamma \backslash X^{+}=S
$$

is called a generalized Hecke operator on $S$ and is denoted by $T_{\varphi}$.

(b) The generalized Hecke operator associated to an inner automorphism $\operatorname{int}(p): p^{\prime} \mapsto p p^{\prime} p^{-1}$ for an element $p \in P(\mathbb{Q})^{+}$is called a (usual) Hecke operator on $S$ and is denoted by $T_{p}$.

Here $\Gamma \cap \varphi^{-1}(\Gamma)$ is again a congruence subgroup of $P(\mathbb{Q})^{+}$, and so both morphisms in 3.1 (a) are finite (possibly ramified) coverings. Thus Hecke operators can be viewed as finite multivalued functions from $S$ to itself in the following sense.

Definition 3.2. For any $T_{\varphi}$ as in Definition 3.1 (a) and any subset $Z \subset S$, the subset

$$
T_{\varphi}(Z):=[\varphi]\left([\mathrm{id}]^{-1}(Z)\right)
$$

is called the translate of $Z$ under $T_{\varphi}$. We also abbreviate $T_{\varphi}(s):=T_{\varphi}(\{s\})$.

By varying the Hecke operator we obtain the following notion.

Proposition-Definition 3.3. Fix a point $s \in S$.

(a) The union of $T_{\varphi}(s)$ for all automorphisms $\varphi$ of $\left(P, X^{+}\right)$is called the generalized Hecke orbit of $s$.

(b) The union of $T_{p}(s)$ for all $p \in P(\mathbb{Q})^{+}$is called the (usual) Hecke orbit of $s$.

Remark 3.4. Using the fact that $P(\mathbb{Q})^{+} \subset P(\mathbb{R})^{+}$is dense, one easily shows that every Hecke orbit, and hence also every generalized Hecke orbit, is Zariski dense. It is dense for the analytic topology if $U=1$.

Remark 3.5. Edixhoven and Yafaev [15] define generalized Hecke orbits differently. They fix a faithful representation $\rho: P \hookrightarrow \mathrm{GL}(V)$ and say that $x^{\prime} \in X^{+}$is equivalent to $x \in X^{+}$if and only if the associated rational Hodge structures on $V$ are isomorphic. The set of all $\left[x^{\prime}\right]$ thus obtained is their generalized Hecke orbit of $[x]$. (Yafaev [51] weakens this condition further by requiring only that the Mumford-Tate groups are isomorphic.) 
Both definitions have their merits, but the relation between them is delicate. We restrict ourselves to the following result.

Proposition 3.6. If $P$ is reductive, every generalized Hecke orbit in the sense of Proposition-Definition 3.3 (a) is contained in a finite union of generalized Hecke orbits in the sense of Remark 3.5.

Proof. Consider any automorphism $\varphi$ of $\left(P, X^{+}\right)$. Let $\bar{\varphi}$ denote the induced automorphism of the torus $\bar{P}:=P / P^{\text {der }}$, and let $\bar{x}$ denote the composite homomorphism $\mathbb{S}_{\mathbb{C}} \stackrel{x}{\rightarrow} P_{\mathbb{C}} \rightarrow \bar{P}_{\mathbb{C}}$ for any $x \in X^{+}$. Then the equation $\bar{\varphi} \circ \bar{x}=\bar{x}$ means that the image of $\bar{x}$ is contained in the kernel of the homomorphism $\bar{P} \rightarrow \bar{P}, \bar{p} \mapsto \bar{\varphi}(\bar{p}) / \bar{p}$. By Axiom (vi) of Definition 2.1 this kernel must therefore be equal to $\bar{P}$. Thus $\bar{\varphi}$ is the identity, and hence the restriction of $\varphi$ to the identity component of the center of $P$ is the identity. On the other hand the outer automorphism group of $P^{\text {der }}$ over $\overline{\mathbb{Q}}$ is finite. Thus to prove the proposition we may restrict ourselves to generalized Hecke operators coming from automorphisms $\varphi$ of $P$ which become inner automorphisms over $\overline{\mathbb{Q}}$.

Let $\rho: P \hookrightarrow \operatorname{GL}(V)$ be the given faithful representation. Then any automorphism $\varphi$ as above fixes the isomorphism class of $\rho$ over $\overline{\mathbb{Q}}$. Thus it also fixes the isomorphism class over $\mathbb{Q}$; hence it is induced by conjugation with an element of $\mathrm{GL}(V)$. But every such automorphism induces an isomorphism of rational Hodge structures on $V$. Thus it preserves the generalized Hecke orbit in the sense of 3.5 , and the proposition follows.

\section{Special and weakly special subvarieties}

In this section we analyze the following class of subvarieties of connected mixed Shimura varieties. Since special subvarieties can be described as connected components of loci of Hodge classes, they are also called 'subvarieties of Hodge type'.

\section{Definition 4.1.}

(a) The image of any Shimura morphism $T \stackrel{[i]}{\longrightarrow} S$ is called a connected mixed Shimura subvariety of $S$, or a special subvariety of $S$.

(b) Consider any Shimura morphisms $T^{\prime} \stackrel{[\varphi]}{\longleftarrow} T \stackrel{[i]}{\longrightarrow} S$ and any point $t^{\prime} \in T^{\prime}$. Then any irreducible component of $[i]\left([\varphi]^{-1}\left(t^{\prime}\right)\right)$ is called a weakly special subvariety of $S$.

Proposition 4.2. Any special subvariety is a weakly special subvariety.

Proof. Let $T_{1}=\left\{t_{1}\right\}$ be the connected Shimura variety associated to the trivial algebraic group. Then for every connected mixed Shimura variety $T$ there exists a unique Shimura morphism $[\varphi]: T \rightarrow T_{1}$, and the proposition follows. 
Proposition 4.3. For any special subvariety of $S$ the Shimura morphism in Definition 4.1 (a) can be chosen such that the underlying homomorphism of algebraic groups $i$ is injective. Then in particular [i] is a Shimura immersion.

Proof. Direct consequence of Proposition 2.8 and Fact 2.6 (b).

Proposition 4.4. For any weakly special subvariety of $S$ the Shimura morphisms in Definition 4.1 (b) can be chosen such that

(a) the underlying homomorphism of algebraic groups $\varphi$ is surjective, and its kernel is connected and possesses no non-trivial torus quotient, and (b) the underlying homomorphism of algebraic groups $i$ is injective.

Then in particular $[\varphi]$ is a Shimura submersion and $[i]$ a Shimura immersion.

Proof. Consider Shimura morphisms $T^{\prime} \stackrel{[\varphi]}{\longleftarrow} T \stackrel{[i]}{\longrightarrow} S$ associated to morphisms of connected mixed Shimura data

$$
\left(Q^{\prime}, Y^{\prime+}\right) \stackrel{\varphi}{\longleftarrow}\left(Q, Y^{+}\right) \stackrel{i}{\longrightarrow}\left(P, X^{+}\right),
$$

and let $Z$ be an irreducible component of $[i]\left([\varphi]^{-1}\left(t^{\prime}\right)\right)$ for some $t^{\prime} \in T^{\prime}$. By Proposition 2.8 we can factor $[\varphi]=\left[i^{\prime}\right] \circ[\psi] \circ[\pi]=\left[i^{\prime} \circ \psi\right] \circ[\pi]$, where $\pi$ is surjective, and $\operatorname{Ker}(\pi)$ is connected and possesses no non-trivial torus quotient, and the identity component of $\operatorname{Ker}\left(i^{\prime} \circ \psi\right)$ is a torus. The last property together with Fact 2.6 (a) implies that $\left[i^{\prime} \circ \psi\right]$ is a finite morphism. It follows that every irreducible component of a fiber of $[\varphi]$ is also an irreducible component of a fiber of $[\pi]$. Since $Z$ is the image under $[i]$ of such an irreducible component, we may replace $[\varphi]$ by $[\pi]$ without changing $Z$. We may therefore assume that $\varphi$ already satisfies the condition (a).

Set $\bar{Q}:=Q / \operatorname{Ker}(i)$ and $\bar{Q}^{\prime}:=Q^{\prime} / \varphi(\operatorname{Ker}(i))$. Then we have a commutative diagram of homomorphisms

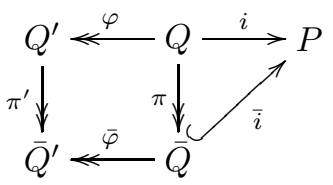

where $\bar{i}$ is injective and $\bar{\varphi}$ also satisfies (a). As in the proof of Proposition 2.8 these homomorphisms induce morphisms of connected mixed Shimura data

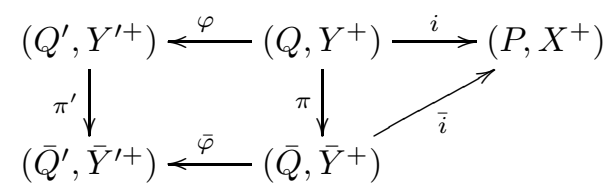

and hence Shimura morphisms 


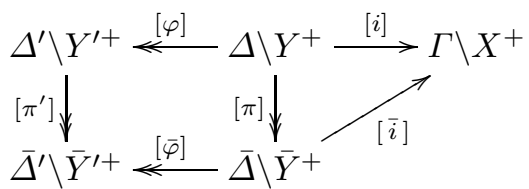

for suitable congruence subgroups. The commutativity of these diagrams implies that

$$
[\pi]\left([\varphi]^{-1}\left(t^{\prime}\right)\right) \subset[\bar{\varphi}]^{-1}\left(\left[\pi^{\prime}\right]\left(t^{\prime}\right)\right) .
$$

On the other hand let $U \triangleleft Q$ be the subgroup of weight -2 from Definition 2.1 (a) and let $d$ be the dimension of any orbit of $\operatorname{Ker}(\varphi)(\mathbb{R}) \cdot(U \cap \operatorname{Ker}(\varphi))(\mathbb{C})$ on $\bar{Y}^{+}$. Since $\bar{Q}^{\prime} \cong \bar{Q} / \pi(\operatorname{Ker}(\varphi))$, both sides of the above inclusion are equidimensional of dimension equal to $d$. Thus every irreducible component of $[\pi]\left([\varphi]^{-1}\left(t^{\prime}\right)\right)$ is also an irreducible component of $[\bar{\varphi}]^{-1}\left(\left[\pi^{\prime}\right]\left(t^{\prime}\right)\right)$. Since $Z$ is the image under $[\bar{i}]$ of such an irreducible component, we may replace $[\varphi],[i]$ by $[\bar{\varphi}],[\bar{i}]$, and the proposition follows.

Remark 4.5. There are two typical examples of weakly special subvarieties. For the first consider three connected mixed Shimura varieties $T_{1}, T_{2}, T$, and a Shimura immersion $[i]: T_{1} \times T_{2} \rightarrow T$. Then for any point $t_{2} \in T_{2}$ the subvariety $Z:=[i]\left(T_{1} \times\left\{t_{2}\right\}\right)$ is weakly special in $T$. When all three Shimura varieties are pure, the subvarieties of $T$ obtained in this way are precisely all totally geodesic irreducible subvarieties of $T$ by a theorem of Moonen [29, II $3.1]$.

The other typical example is discussed in the following proposition.

Proposition 4.6. Let $[\pi]: A \rightarrow S$ be a Shimura epimorphism as in (2.11), so that the fiber $A_{s}$ over any point $s \in S$ is an abelian variety. Then the weakly special subvarieties of $A$ that are contained in $A_{s}$ are precisely the translates of abelian subvarieties of $A_{s}$.

Proof. First let $Z$ be a weakly special subvariety of $A$. Then by definition there exist Shimura morphisms

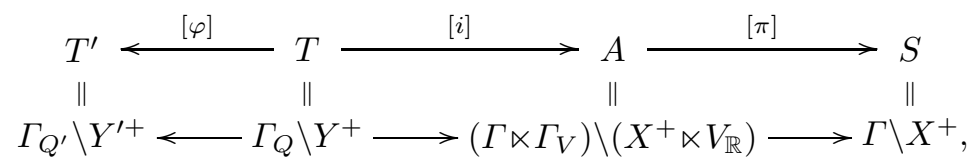

associated to morphisms of connected mixed Shimura data

$$
\left(Q^{\prime}, Y^{\prime+}\right) \stackrel{\varphi}{\longleftarrow}\left(Q, Y^{+}\right) \stackrel{i}{\longrightarrow}\left(P \ltimes V_{\mathrm{alg}}, X^{+} \ltimes V_{\mathbb{R}}\right) \stackrel{\pi}{\longrightarrow}\left(P, X^{+}\right),
$$

such that $Z$ is an irreducible component of $[i]\left([\varphi]^{-1}\left(t^{\prime}\right)\right)$ for some point $t^{\prime} \in T^{\prime}$. By Proposition 4.4 we may assume that $\varphi$ is surjective, that $K:=\operatorname{Ker}(\varphi)$ is 
connected without a non-trivial torus quotient, and that $i$ is injective. Observe that any irreducible component $Z^{+} \subset X^{+} \ltimes V_{\mathbb{R}}$ of the preimage of $Z \subset A$ is invariant under the action of the identity component $i\left(K(\mathbb{R})^{\circ}\right)$.

Assume that $Z \subset A_{s}$. Then $[\pi](Z)=\{s\}$ implies that $\pi$ maps $Z^{+}$to a point in $X^{+}$. Thus $\pi \circ i\left(K(\mathbb{R})^{\circ}\right)$ fixes that point. By the Axioms (ii-iv) of Definition 2.1 this implies that $\pi \circ i\left(K(\mathbb{R})^{\circ}\right)$ is compact. In particular $\pi \circ i(K)$ is reductive. As a quotient of $K$ it is also connected without a non-trivial torus quotient; hence it is connected semisimple. Thus $(\pi \circ i(K))^{\text {ad }}$ is a connected semisimple quotient of $Q$ of compact type, so by Definition 2.1 (vi) it must be trivial. Altogether this implies that $\pi \circ i(K)=1$.

This means that $i(K) \subset \operatorname{Ker}(\pi)=V_{\text {alg }}$. Since $V_{\text {alg }}$ is of Hodge weight -1 , so is $K$, and so all fibers of $Y^{+} \rightarrow Y^{\prime+}$ are orbits under $K(\mathbb{R})$. Thus $Z^{+}$is an orbit under $i(K(\mathbb{R}))$. Its image $Z \subset A_{s} \cong \Gamma_{V} \backslash V_{\mathbb{R}}$ is therefore a translate of a connected closed subgroup. Being a complex analytic subvariety, it must be a translate of an abelian subvariety, as desired.

Conversely, let $Z$ be a translate of an abelian subvariety $B \subset A_{s}$. Recall that $A_{s} \cong \Gamma_{V} \backslash V_{\mathbb{R}}$ with the complex structure determined by any preimage $x \in X^{+}$of $s$. Thus $B \cong\left(\Gamma_{V} \cap V^{\prime}\right) \backslash V_{\mathbb{R}}^{\prime}$ for some $\mathbb{Q}$-subspace $V^{\prime} \subset V$, and since $B$ is also a complex subvariety, the action of $\mathbb{S}$ on $V_{\mathbb{R}}$ determined by $x$ must leave $V_{\mathbb{R}}^{\prime}$ invariant. In other words $x \in \operatorname{Hom}\left(\mathbb{S}_{\mathbb{C}}, P_{\mathbb{C}}\right)$ factors through $P_{\mathbb{C}}^{\prime}$ for $P^{\prime}:=\operatorname{Stab}_{P}\left(V^{\prime}\right)$.

Let $Q \subset P^{\prime}$ be the smallest connected algebraic subgroup defined over $\mathbb{Q}$ such that $x$ factors through $Q_{\mathbb{C}}$. (This is the Mumford-Tate group of $x$.) Using the Axioms of Definition 2.1 for $\left(P, X^{+}\right)$one easily checks that there is a unique connected mixed Shimura datum $\left(Q, Y^{+}\right)$with $x \in Y^{+}$, such that the inclusion defines a morphism $\left(Q, Y^{+}\right) \rightarrow\left(P, X^{+}\right)$. Consider the induced morphisms of connected mixed Shimura data

$$
\left(Q \ltimes\left(V / V^{\prime}\right)_{\mathrm{alg}}, Y^{+} \ltimes\left(V / V^{\prime}\right) \mathbb{R}\right) \stackrel{\varphi}{\longleftarrow}\left(Q \ltimes V_{\mathrm{alg}}, Y^{+} \ltimes V_{\mathbb{R}}\right) \stackrel{i}{\longrightarrow}\left(P \ltimes V_{\mathrm{alg}}, X^{+} \ltimes V_{\mathbb{R}}\right)
$$

and of the associated connected mixed Shimura varieties. In the fibers above the point $s=[x] \in \Gamma \backslash X^{+}$these maps are simply

$$
A_{s} / B \stackrel{[\varphi]}{\longleftarrow} A_{s} \stackrel{\text { id }}{\longrightarrow} A_{s} .
$$

Thus $Z$, being a translate of $B$, is also a fiber of $[\varphi]$, and therefore a weakly special subvariety according to Definition 4.1, as desired.

The following fact shows that the conclusion in Conjecture 1.6 cannot be strengthened.

Proposition 4.7. For any weakly special subvariety $Z \subset S$ and any point $s \in Z$, the intersection of $Z$ with the Hecke orbit of $s$ is Zariski dense in $Z$. 
Proof. Consider Shimura morphisms $T^{\prime} \stackrel{[\varphi]}{\longleftarrow} T \stackrel{[i]}{\longrightarrow} S$ associated to morphisms of connected mixed Shimura data

$$
\left(Q^{\prime}, Y^{\prime+}\right) \stackrel{\varphi}{\longleftarrow}\left(Q, Y^{+}\right) \stackrel{i}{\longrightarrow}\left(P, X^{+}\right),
$$

and let $Z$ be an irreducible component of $[i]\left([\varphi]^{-1}\left(t^{\prime}\right)\right)$ for a point $t^{\prime} \in T^{\prime}$. Then $Z=[i](\tilde{Z})$ for an irreducible component $\tilde{Z}$ of $[\varphi]^{-1}\left(t^{\prime}\right)$. For all $t \in \tilde{Z}$, the definition of Hecke operators implies that $[i]\left(T_{q}(t)\right) \subset T_{i(q)}([i](t))$ for all $q \in Q(\mathbb{Q})^{+}$. Thus $[i]$ maps the Hecke orbit of $t$ in $T$ into the Hecke orbit of $[i](t)$ in $S$. It therefore suffices to prove that the intersection of $\tilde{Z}$ with the Hecke orbit of $t$ is Zariski dense in $\tilde{Z}$.

Write $T=\Delta \backslash Y^{+}$and $t=[y]$ for $y \in Y^{+}$. Set $K:=\operatorname{Ker}(\varphi)$. Using the Hecke operators $T_{k}$ for all $k \in K(\mathbb{Q}) \cap K(\mathbb{R})^{\circ}$ one finds that $\Delta \backslash \Delta(K(\mathbb{Q}) \cap$ $\left.K(\mathbb{R})^{\circ}\right) y$ is a subset of the Hecke orbit of $t$ that is contained in $[\varphi]^{-1}\left(t^{\prime}\right)$. Its closure for the analytic topology contains the connected real analytic subset $\Delta \backslash \Delta K(\mathbb{R})^{\circ} y$. In particular this subset is contained in $\tilde{Z}$. Moreover, let $U \triangleleft Q$ be the subgroup of weight -2 from Definition 2.1 (a). Then any $K(\mathbb{R})^{\circ}$-invariant complex analytic subspace of $Y^{+}$is also invariant under $K(\mathbb{R})^{\circ} \cdot(U \cap K)(\mathbb{C})$. Since this group also acts transitively on the fibers of $Y^{+} \rightarrow Y^{++}$, we deduce that the Zariski closure of $\Delta \backslash \Delta K(\mathbb{R})^{\circ} y$ is equal to $\tilde{Z}$. Altogether this shows that the Hecke orbit of $t$ in $T$ contains a Zariski dense subset of $\tilde{Z}$, as desired.

Remark 4.8. For a usual, non-connected, Shimura variety $\tilde{S}$ the Definition 4.1 of special and weakly special subvarieties must be modified slightly, for a technical reason. The problem is that the images of Shimura morphisms may not meet all components of $\tilde{S}$. As a remedy one replaces $[i]$ by $T_{p} \circ[i]$ in Definition 4.1 for all Hecke operators $T_{p}$ on $\tilde{S}$. The same applies to Definition 4.10 below.

Remark 4.9. One easily shows that any irreducible component of an intersection of special subvarieties of $S$ is a special subvariety. By noetherian induction it follows that every irreducible subvariety of $S$ is contained in a unique smallest special subvariety. The analogous assertions hold for weakly special subvarieties; the proofs are left to the reader.

Definition 4.10. $A$ point $x \in X^{+}$and its image in $S$ are called special if the homomorphism $x: \mathbb{S}_{\mathbb{C}} \rightarrow P_{\mathbb{C}}$ factors through $H_{\mathbb{C}}$ for a torus $H \subset P$ defined over $\mathbb{Q}$.

Thus $x \in X^{+}$is special if and only if it is the image of a morphism of connected mixed Shimura data $\left(H, Y^{+}\right) \rightarrow\left(P, X^{+}\right)$where $H$ is a torus. Correspondingly a point $s \in S$ is special if and only if $\{s\}$ is a special subvariety of dimension 0. Clearly the image of any special point under any Shimura morphism is again a special point. 
Remark 4.11. For every point $s \in S$ the set $\{s\}$ is a fiber of the identity morphism id: $S \rightarrow S$ and therefore a weakly special subvariety according to Definition 4.1 (b). Thus a concept of 'weakly special points' analogous to that of 'special points' would not be very useful.

Remark 4.12. A point on the connected Shimura variety associated to the pair $\left(\mathrm{CSp}_{2 g, \mathbb{Q}}, \mathcal{H}_{2 g}\right)$ from Example 2.12 is special if and only if the associated abelian variety has complex multiplication. Here a non-simple abelian variety is said to have complex multiplication if and only if every simple constituent has complex multiplication.

Remark 4.13. Let $V$ and $A \rightarrow S$ be as in Construction 2.9. Consider a special point $y \in X^{+} \ltimes V_{\mathbb{R}}$, corresponding to a homomorphism $y$ : $\mathbb{S}_{\mathbb{C}} \rightarrow H_{\mathbb{C}} \subset$ $P_{\mathbb{C}} \ltimes V_{\text {alg, } \mathbb{C}}$ for a torus $H \subset P \ltimes V_{\text {alg }}$ defined over $\mathbb{Q}$. Then $H$ can be conjugated into $P$ by an element of $V_{\text {alg }}(\mathbb{Q})=V$. The isomorphism $X^{+} \times V_{\mathbb{R}} \cong X^{+} \ltimes V_{\mathbb{R}}$ from (2.10) shows that this conjugation induces translation on $V_{\mathbb{R}}$. Thus it follows that the special points in $A$ are precisely the torsion points in the fibers $A_{s}$ over all special points $s \in S$. Compare also Proposition 5.1 (a).

Proposition 4.14. Every special subvariety of $S$ contains a Zariski dense subset of special points.

Proof. Since any Shimura morphism maps special points to special points, it suffices to show that the set of special points of every connected mixed Shimura variety is Zariski dense. This is well-known, for example by [33, 11.7].

Proposition 4.15. Every weakly special subvariety of $S$ that contains a special point is a special subvariety.

Proof. Consider Shimura morphisms $T^{\prime} \stackrel{[\varphi]}{\longleftarrow} T \stackrel{[i]}{\longrightarrow} S$ associated to morphisms of connected mixed Shimura data

$$
\left(Q^{\prime}, Y^{\prime+}\right) \stackrel{\varphi}{\longleftarrow}\left(Q, Y^{+}\right) \stackrel{i}{\longrightarrow}\left(P, X^{+}\right),
$$

and let $Z$ be an irreducible component of $[i]\left([\varphi]^{-1}\left(t^{\prime}\right)\right)$ for some $t^{\prime} \in T^{\prime}$. By Proposition 4.4 we may assume that $i$ is injective. Let $y \in Y^{+}$be a point whose image in $S$ is a special point in $Z$. Then the homomorphism $i \circ y: \mathbb{S}_{\mathbb{C}} \rightarrow P_{\mathbb{C}}$ factors through a torus defined over $\mathbb{Q}$. Since $i$ is injective, this implies that $y: \mathbb{S}_{\mathbb{C}} \rightarrow Q_{\mathbb{C}}$ factors through a torus defined over $\mathbb{Q}$. Let $H \subset Q$ be such a torus. Let $K$ denote the identity component of $\operatorname{Ker}(\varphi)$ and consider the subgroup $Q_{1}:=K \cdot H \subset Q$. Then there exists a unique connected mixed Shimura datum $\left(Q_{1}, Y_{1}^{+}\right)$such that $y \in Y_{1}^{+}$, and the associated special subvariety $T_{1} \subset T$ is an irreducible component of $[\varphi]^{-1}\left(t^{\prime}\right)$. It follows that $Z$ is the image of $T_{1}$ and hence a special subvariety, as desired.

Remark 4.16. Consider the weakly special subvariety $Z:=[i]\left(T_{1} \times\left\{t_{2}\right\}\right) \subset T$ from Remark 4.5. The preceding results imply that $Z$ is special if and only if $t_{2}$ is a special point. (Compare Moonen [29, II 3.1].) 
Remark 4.17. Let $V$ and $A \rightarrow S$ be again as in Construction 2.9. The preceding results imply that a fiber $A_{s}$ contains a special subvariety if and only if the base point $s \in S$ is special. Moreover, if $s$ is special, then the special subvarieties contained in $A_{s}$ are precisely the translates of abelian subvarieties by torsion points.

Theorem 4.18 (Edixhoven-Yafaev). Conjecture 1.6 is true when $S$ is a pure Shimura variety, $s \in S$ is a special point, and $Z \subset S$ is a curve.

Proof. Under the given assumptions, if $Z \cap \Lambda$ is Zariski dense in $Z$ for some generalized Hecke orbit $\Lambda$ in the sense of Proposition-Definition 3.3 (a), Proposition 3.6 implies that the same is true for some generalized Hecke orbit in the sense of Remark 3.5. By Edixhoven and Yafaev [15] it thus follows that $Z$ is a special subvariety of $S$.

\section{Relation with the Mordell-Lang conjecture}

In this section we show that Conjecture 1.6 for subvarieties of an abelian fiber $A_{s}$ above a pure Shimura variety is equivalent to the Mordell-Lang conjecture. The argument is based on a description of the generalized Hecke orbit of a point in $A_{s}$ as a point on the ambient connected mixed Shimura variety.

We use the notation of Construction 2.9. Thus $\left(P, X^{+}\right)$is a connected Shimura datum and $V$ is a representation of $P$ of Hodge type $\{(-1,0),(0,-1)\}$, as in Example 2.12. To the extended connected mixed Shimura datum $\left(P \ltimes V_{\text {alg }}, X^{+} \ltimes V_{\mathbb{R}}\right)$ and suitable congruence subgroups is associated a Shimura epimorphism

$$
A:=\left(\Gamma \ltimes \Gamma_{V}\right) \backslash\left(X^{+} \ltimes V_{\mathbb{R}}\right) \stackrel{[\pi]}{\longrightarrow} \Gamma \backslash X^{+}=: S .
$$

Fix a point $s \in S$. Then the fiber $A_{s}:=[\pi]^{-1}(s)$ is an abelian variety isomorphic to $\Gamma_{V} \backslash V_{\mathbb{R}}$ with a complex structure defined by $s$. Its torsion subgroup is $A_{s, \text { tor }}=\Gamma_{V} \backslash V$.

Proposition 5.1. Let $\varpi$ denote the projection $V_{\mathbb{R}} \rightarrow \Gamma_{V} \backslash V_{\mathbb{R}} \cong A_{s}$. Let $v$ be any point in $V_{\mathbb{R}}$, and set $a:=\varpi(v)$.

(a) The Hecke orbit of a as a point on A contains the subset

$$
\varpi\left(\left\{v+v^{\prime} \mid v^{\prime} \in V\right\}\right)=\varpi(v)+A_{s, \text { tor }} \subset A_{s} .
$$

(b) The generalized Hecke orbit of a as a point on A contains the subset

$$
\varpi\left(\left\{g(v)+v^{\prime} \mid g \in \operatorname{Aut}_{P}(V), v^{\prime} \in V\right\}\right) \subset A_{s} .
$$


(c) If $\left(P, X^{+}\right)$is pure, the intersection with $A_{s}$ of the generalized Hecke orbit of a as a point on $A$ is contained in the subset

$$
\left\{b \in A_{s} \mid \exists n \in \mathbb{Z} \backslash\{0\}: \exists h \in \operatorname{End}\left(A_{s}\right): n b=h(a)\right\} .
$$

Proof. First consider the inner automorphisms of $P$ induced by elements of $V$. Via the isomorphism $X^{+} \times V_{\mathbb{R}} \cong X^{+} \ltimes V_{\mathbb{R}}$ from (2.10) they act by translation on $V_{\mathbb{R}}$. Thus all translations by $V$ on $\Gamma_{V} \backslash V_{\mathbb{R}}$ are induced by usual Hecke operators, proving (a).

For (b) we attach to $g \in \operatorname{Aut}_{P}(V)$ the automorphism $(p, v) \mapsto(p, g(v))$ of $P \ltimes V_{\text {alg. }}$. It is the identity on $P$, and therefore maps $X^{+}$and hence $X^{+} \ltimes V_{\mathbb{R}}$ to itself. Thus it defines a generalized Hecke operator on $A$. Via the isomorphism $X^{+} \times V_{\mathbb{R}} \cong X^{+} \ltimes V_{\mathbb{R}}$ from (2.10) it acts on the latter by $(x, v) \mapsto(x, g(v))$. Thus it acts by $g$ on the fibers $V_{\mathbb{R}}$. Since $g \in \operatorname{Aut}_{P}(V)$ is arbitrary, and translations by $V$ are already induced by usual Hecke operators, this proves (b).

For (c) consider any automorphism $\varphi$ of the connected mixed Shimura datum $\left(P \ltimes V_{\text {alg }}, X^{+} \ltimes V_{\mathbb{R}}\right)$. By assumption $P$ is reductive; hence $V_{\text {alg }}$ is the unipotent radical of $P \ltimes V_{\text {alg }}$ and is therefore mapped to itself under $\varphi$. On the other hand, since all Levi decompositions of $P \ltimes V_{\text {alg }}$ are conjugate under $V$, there exists $v^{\prime} \in V$ such that $\psi:=\operatorname{int}\left(v^{\prime}\right)^{-1} \circ \varphi$ maps $P$ to itself. The generalized Hecke operator $T_{\varphi}$ on $A$ is then the composite of the generalized Hecke operator $T_{\psi}$ with the translation by $\varpi\left(v^{\prime}\right)$ along the abelian fibers. In the fiber above $s$, the operator $T_{\psi}$ consists of finitely many diagrams of the form

$$
A_{s} \longleftarrow A_{s^{\prime}}^{\prime} \longrightarrow A_{s}
$$

for finite morphisms between abelian varieties. By construction $T_{\psi}$ preserves the zero sections; hence these morphisms are isogenies. Therefore $T_{\psi}(a) \cap$ $A_{s}$ is contained in the indicated subset. Since this subset is invariant under translation by $\varpi\left(v^{\prime}\right) \in A_{s, \text { tor }}$, the assertion follows.

Theorem 5.2. In the situation above, if $\left(P, X^{+}\right)$is pure, Conjecture 1.6 for subvarieties of $A_{s}$ follows from the Mordell-Lang conjecture.

Proof. Let $a \in A_{s}$ be any point and $\Lambda$ its generalized Hecke orbit as a point on $A$. Let $Z \subset A_{s}$ be an irreducible closed algebraic subvariety such that $Z \cap \Lambda$ is Zariski dense in $Z$. Then Proposition 5.1 (c) implies that the intersection of $Z$ with the division group of $\operatorname{End}\left(A_{s}\right) a \subset A_{s}$ is Zariski dense in $Z$. Since $\operatorname{End}\left(A_{s}\right)$ is a finitely generated $\mathbb{Z}$-module, the Mordell-Lang Conjecture 1.4 implies that $Z$ is a translate of an abelian subvariety of $A_{s}$. Thus by Proposition 4.6 it is a weakly special subvariety of $A$, as desired.

To prove the converse, for any point $a \in A_{s}$ we consider the set

$$
\Lambda_{a}^{*}:=\left\{b \in A_{s} \mid \exists m, n \in \mathbb{Z} \backslash\{0\}: n b=m a\right\} .
$$


If $a=\varpi(v)$ for $v \in V_{\mathbb{R}}$, we find that $\Lambda_{a}^{*}=\varpi\left(\mathbb{Q}^{*} \cdot v+V\right)$. Since the scalars $\mathbb{Q}^{*}$ are contained in $\operatorname{Aut}_{P}(V)$, Proposition 5.1 (b) shows that $\Lambda_{a}^{*}$ lies inside in the generalized Hecke orbit of $a$. Using this one can already show that Conjecture 1.6 implies the Mordell-Lang conjecture for subgroups $\Lambda$ of $\mathbb{Q}$-rank $\leq 1$. For arbitrary rank we need one more technical lemma.

Lemma 5.3. For any elements $a_{1}, \ldots, a_{r} \in A_{s}$ satisfying $a_{1}+\cdots+a_{r}=0$, we have

$$
\left\{b \in A_{s} \mid \exists n \in \mathbb{Z}^{>0}: n b \in \mathbb{Z} a_{1}+\cdots+\mathbb{Z} a_{r}\right\}=\Lambda_{a_{1}}^{*}+\cdots+\Lambda_{a_{r}}^{*} .
$$

Proof. The inclusion ' $\supset$ ' is obvious. To prove ' $\subset$ ' consider an element $b$ of the left-hand side. Then there exist integers $n>0$ and $n_{1}, \ldots, n_{r}$ such that $n b=\sum_{i} n_{i} a_{i}$. The assumption implies that for any integer $m$ we then also have $n b=\sum_{i}\left(n_{i}+m\right) a_{i}$. Since we can choose $m$ so that all the coefficients $n_{i}+m$ are non-zero, this equation shows that $b$ lies in the right-hand side, as desired.

Theorem 5.4. Conjecture 1.6 implies the Mordell-Lang conjecture.

Proof. Every abelian variety over $\mathbb{C}$ is isomorphic to some $A_{s}$ as above by Example 2.12. So consider a finitely generated subgroup $\Lambda_{0} \subset A_{s}$. Fix generators $a_{1}, \ldots, a_{r-1}$ of $\Lambda_{0}$ and set $a_{r}:=-a_{1}-\cdots-a_{r-1}$. Let $A_{s}^{r}$ be the product of $r$ copies of $A_{s}$, and let $\Sigma: A_{s}^{r} \rightarrow A_{s}$ denote the summation map $\left(b_{1}, \ldots, b_{r}\right) \mapsto b_{1}+\cdots+b_{r}$. Then Lemma 5.3 asserts that the division group $\Lambda$ of $\Lambda_{0}$ satisfies

$$
\Lambda=\Lambda_{a_{1}}^{*}+\cdots+\Lambda_{a_{r}}^{*}=\Sigma\left(\Lambda_{a_{1}}^{*} \times \cdots \times \Lambda_{a_{r}}^{*}\right) .
$$

Let $V^{r}$ be the direct sum of $r$ copies of $V$ as a representation of $P$. Then the connected mixed Shimura variety associated to $P \ltimes V_{\text {alg }}^{r}$,

$$
A^{r}:=\left(\Gamma \ltimes \Gamma_{V}^{r}\right) \backslash\left(X^{+} \ltimes V_{\mathbb{R}}^{r}\right),
$$

is the $r$-fold fiber product of $A$ with itself over $S$, and its fiber over $s$ is $A_{s}^{r}$. Let tuples $\left(u_{1}, \ldots, u_{r}\right)$ of elements of $\mathbb{Q}^{*}$ act on $V^{r}$ by scalar multiplication on the respective direct summands. This defines $P$-equivariant automorphisms of $V^{r}$; hence Proposition 5.1 (b) implies that the generalized Hecke orbit of $\left(a_{1}, \ldots, a_{r}\right) \in A_{s}^{r}$ as a point on the connected mixed Shimura variety $A^{r}$ contains $\Lambda_{a_{1}}^{*} \times \cdots \times \Lambda_{a_{r}}^{*}$.

Let $Z \subset A$ be an irreducible closed algebraic subvariety such that $Z \cap \Lambda$ is Zariski dense in $Z$. Then with

$$
\tilde{\Lambda}:=\Sigma^{-1}(Z) \cap\left(\Lambda_{a_{1}}^{*} \times \cdots \times \Lambda_{a_{r}}^{*}\right)
$$

we find that 


$$
\Sigma(\tilde{\Lambda})=Z \cap \Sigma\left(\Lambda_{a_{1}}^{*} \times \cdots \times \Lambda_{a_{r}}^{*}\right)=Z \cap \Lambda
$$

is Zariski dense in $Z$. Let $\tilde{Z} \subset A_{s}^{r}$ denote the Zariski closure of $\tilde{\Lambda}$. Since taking Zariski closures commutes with taking images under proper morphisms, we deduce that $\Sigma(\tilde{Z})=Z$. Choose an irreducible component $Z^{\prime}$ of $\tilde{Z}$ such that $\Sigma\left(Z^{\prime}\right)=Z$. Then by construction the intersection of $Z^{\prime}$ with the generalized Hecke orbit of $\left(a_{1}, \ldots, a_{r}\right)$ is Zariski dense in $Z^{\prime}$. Thus Conjecture 1.6 for the connected mixed Shimura variety $A^{r}$ implies that $Z^{\prime}$ is weakly special. Therefore $Z=\Sigma\left(Z^{\prime}\right)$ is weakly special, and so by Proposition 4.6 it is a translate of an abelian subvariety of $A_{s}$, as desired.

Remark 5.5. The above results generalize easily to semi-abelian varieties, on the basis of Remark 2.13 and a suitable generalization of Proposition 4.6.

\section{Hodge and Galois generic points}

The first notion is opposite to that of special points.

Definition 6.1. A point $x \in X^{+}$and its image in $S$ are called Hodge generic if the homomorphism $x: \mathbb{S}_{\mathbb{C}} \rightarrow P_{\mathbb{C}}$ does not factor through $P_{\mathbb{C}}^{\prime}$ for any proper algebraic subgroup $P^{\prime} \subset P$ defined over $\mathbb{Q}$.

Remark 6.2. The Axiom (vi) of Definition 2.1 implies that for any fixed proper algebraic subgroup $P^{\prime} \subset P$ defined over $\mathbb{Q}$, the set of $x \in X^{+}$that factor through $P_{\mathbb{C}}^{\prime} \subset P_{\mathbb{C}}$ is a proper analytic subspace. Since there are only countably many possibilities for $P^{\prime}$, their union is a subset of the first category of Baire. In particular its measure is zero. In this sense, 'most' points in $X^{+}$ and $S$ are Hodge generic.

Next we look at Galois theoretic properties. To clarify the dependence on the congruence subgroup $\Gamma$, we write $S_{\Gamma}:=\Gamma \backslash X^{+}$and $[x]_{\Gamma} \in S_{\Gamma}$ from now on. We have already used the fact that $S_{\Gamma}$ is an algebraic variety over $\mathbb{C}$. But it also possesses a model over a number field, determined by abelian class field theory. Fix any number field $E$ that contains the so-called reflex field of $\left(P, X^{+}\right)$(compare $\left.[33,11.1]\right)$. Then the non-connected mixed Shimura variety corresponding to $\left(P, X^{+}\right)$possesses a so-called canonical model over $E$ by [33, 11.18]. This implies that $S_{\Gamma}$ possesses a natural model over the maximal abelian extension $E_{\mathrm{ab}}$ of $E$. By abuse of notation we denote this model again by $S_{\Gamma}$. By $[33,11.10]$ it is functorial in $\left(P, X^{+}\right)$and $\Gamma$. In particular, for any automorphism $\varphi$ of $\left(P, X^{+}\right)$and any congruence subgroups $\Gamma \subset P(\mathbb{Q})^{+}$and $\Gamma^{\prime} \subset \varphi^{-1}(\Gamma)$, the Shimura morphism $[\varphi]: S_{\Gamma^{\prime}} \rightarrow S_{\Gamma}$ is defined over $E_{\mathrm{ab}}$.

Let $s$ be any $\mathbb{C}$-valued point on $S_{\Gamma}$. Let $K \subset \mathbb{C}$ be any finitely generated extension field of $E_{\mathrm{ab}}$, such that $s$ is defined over $K$. Let $\bar{K}$ denote the algebraic closure of $K$ in $\mathbb{C}$. Then for any congruence subgroup $\Gamma^{\prime} \subset \Gamma$ the Galois group 
$\operatorname{Gal}(\bar{K} / K)$ acts on the inverse image of $s$ under the morphism [id] : $S_{\Gamma^{\prime}} \rightarrow S_{\Gamma}$. When $\Gamma$ is sufficiently small and $\Gamma^{\prime}$ is normal in $\Gamma$, this action corresponds to a continuous homomorphism

$$
\operatorname{Gal}(\bar{K} / K) \longrightarrow \Gamma / \Gamma^{\prime} .
$$

Let $\mathbb{A}_{f}$ denote the ring of finite adèles of $\mathbb{Q}$ and $\bar{\Gamma}$ the closure of $\Gamma$ in $P\left(\mathbb{A}_{f}\right)$. By going to the limit over all $\Gamma^{\prime}$ the above homomorphisms induce a continuous homomorphism

$$
\operatorname{Gal}(\bar{K} / K) \longrightarrow \bar{\Gamma} \subset P\left(\mathbb{A}_{f}\right) .
$$

We denote its image by $\bar{\Gamma}_{s, K}$. Axiom (v) of Definition 2.1 implies that a subgroup of finite index of $\Gamma$ is contained in $P^{\text {der }}(\mathbb{Q})$, where $P^{\text {der }}$ denotes the derived group of $P$. Thus after shrinking $\Gamma$ and extending $K$ we can always achieve $\bar{\Gamma}_{s, K} \subset \bar{\Gamma} \subset P^{\operatorname{der}}\left(\mathbb{A}_{f}\right)$.

Definition 6.3. Let $s$ and $K$ be as above.

(a) $s$ is called Galois generic if $\bar{\Gamma}_{s, K}$ is open in $\bar{\Gamma}$.

(b) $s$ is called strictly Galois generic with respect to $K$ if $\bar{\Gamma}_{s, K}=\bar{\Gamma}$.

Proposition 6.4. The notion 'Galois generic' is independent of the choice of $K$ and $E$ and invariant under taking images and preimages by morphisms induced by automorphisms of $\left(P, X^{+}\right)$, and so in particular by generalized Hecke operators.

Proof. The independence of $K$ follows from the fact that for any finitely generated field extension $K^{\prime}$ of $K$, the natural homomorphism $\operatorname{Gal}\left(\bar{K}^{\prime} / K^{\prime}\right) \rightarrow$ $\operatorname{Gal}(\bar{K} / K)$ has an open image. The invariance under morphisms and generalized Hecke operators results from the construction. For the independence of $E$ we must show that for any finite extension $E^{\prime}$ of $E$, the group $\bar{\Gamma}_{s, K E_{\mathrm{ab}}^{\prime}}$ is open in $\bar{\Gamma}$, if $\bar{\Gamma}_{s, K}$ is open in $\bar{\Gamma}$. (This part of the proof could be formulated more naturally in the framework of non-connected Shimura varieties.)

For this we may assume that $\Gamma$ is sufficiently small, so that it is torsion free and contained in $P^{\text {der }}(\mathbb{Q})$. Next note that $S_{\Gamma}$ already possesses a model $S_{\Gamma, E_{\Gamma}}$ over some subfield $E_{\Gamma} \subset E_{\mathrm{ab}}$ which is finite over $E$. The theory of canonical models of non-connected mixed Shimura varieties yields a continuous homomorphism from the étale fundamental group $\pi_{1, \text { ét }}\left(S_{\Gamma, E_{\Gamma}}, s\right) \rightarrow P\left(\mathbb{A}_{f}\right)$. Similarly, $s$ is defined over a subfield $L \subset K$ that is finitely generated over $E_{\Gamma}$. By the independence of $K$ we may assume that $K=L E_{\mathrm{ab}}$ and $E^{\prime} \subset L$. Setting $K^{\prime}:=K E_{\mathrm{ab}}^{\prime}$, we have $\bar{K}^{\prime}=\bar{K}=\bar{L}$ and hence the inclusions in the upper row of the following commutative diagram. The groups in the lower row are defined as the respective images:

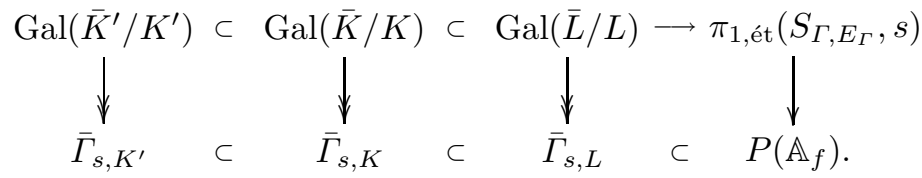


Since $K^{\prime}=K E_{\mathrm{ab}}^{\prime}=L E_{\mathrm{ab}}^{\prime}$ with $E^{\prime} \subset L$, we deduce that $\bar{\Gamma}_{s, K^{\prime}}$ contains the commutator subgroup of $\bar{\Gamma}_{s, L}$.

Assume now that $\bar{\Gamma}_{s, K}$ is open in $\bar{\Gamma}$. Then $\bar{\Gamma}_{s, K}$ contains an open subgroup of $W\left(\mathbb{A}_{f}\right)$, where $W$ is the unipotent radical of $P$. We claim that the commutators of $\bar{\Gamma}_{s, L}$ with any open subgroup of $W\left(\mathbb{A}_{f}\right)$ generate an open subgroup of $W\left(\mathbb{A}_{f}\right)$. To prove this we may replace $W$ by any simple subquotient. If $P^{\text {der }}$ acts non-trivially on this subquotient, then so does any congruence subgroup of $\Gamma$ and the assertion follows. If $P^{\text {der }}$ acts trivially on this subquotient, the induced action of $P / P^{\text {der }}$ must be non-trivial by the Axiom (iii) of Definition 2.1. The action of $\bar{\Gamma}_{s, L}$ on the subquotient is then determined by abelian class field theory applied to the torus $P / P^{\text {der }}$, and the claim follows from a straightforward exercise using the reciprocity law. Together this implies the claim for $W$; hence $\bar{\Gamma}_{s, K^{\prime}}$ contains an open subgroup of $W\left(\mathbb{A}_{f}\right)$.

On the other hand, by strong approximation the image $\bar{\Gamma}_{\mathrm{ss}}$ of $\bar{\Gamma}$ in $\left(P^{\text {der }} / W\right)\left(\mathbb{A}_{f}\right)$ is open in the image of the group of $\mathbb{A}_{f}$-valued points on the simply connected covering of $P^{\text {der }} / W$. From this one deduces that the commutator subgroup of any open subgroup of $\bar{\Gamma}_{\mathrm{ss}}$ is open. Therefore the image of $\bar{\Gamma}_{s, K^{\prime}}$ in $\left(P^{\text {der }} / W\right)\left(\mathbb{A}_{f}\right)$ is open in the image of $\bar{\Gamma}$. Altogether we deduce that $\bar{\Gamma}_{s, K^{\prime}}$ is open in $\bar{\Gamma}$, as desired.

Proposition 6.5. For any $s \in S_{\Gamma}$ the following assertions are equivalent:

(a) $s$ is Galois generic.

(b) There exist a finitely generated extension $K \subset \mathbb{C}$ of $E_{\mathrm{ab}}$, a congruence subgroup $\Gamma^{\prime} \subset \Gamma$, and a point $s^{\prime} \in S_{\Gamma^{\prime}}$ mapping to $s$ under $[\mathrm{id}]: S_{\Gamma^{\prime}} \rightarrow S_{\Gamma}$, such that $s^{\prime}$ is strictly Galois generic with respect to $K$.

(c) Same as (b), but for every sufficiently large $K$, i.e., for every $K$ containing some fixed finitely generated extension $K_{0}$ of $E_{\mathrm{ab}}$.

Proof. The implication (c) $\Rightarrow(\mathrm{b})$ is obvious, and the implication (b) $\Rightarrow(\mathrm{a})$ follows from the equality $\bar{\Gamma}_{s^{\prime}, K}=\bar{\Gamma}_{s, K}$. For the remaining implication (a) $\Rightarrow$ (c) assume that $\bar{\Gamma}_{s, K_{0}}$ is open in $\bar{\Gamma}$ for some finitely generated extension $K_{0}$ of $E_{\mathrm{ab}}$ over which $s$ is defined. Then for every finitely generated extension $K \subset \mathbb{C}$ of $K_{0}$, the group $\bar{\Gamma}_{s, K}$ is open in $\bar{\Gamma}$. Set $\Gamma^{\prime}:=\bar{\Gamma}_{s, K} \cap \Gamma$. Then the construction of $\bar{\Gamma}_{s, K}$ shows that some point $s^{\prime} \in S_{\Gamma^{\prime}}$ above $s$ is fixed by $\operatorname{Gal}(\bar{K} / K)$ and satisfies $\bar{\Gamma}_{s^{\prime}, K}=\bar{\Gamma}_{s, K}=\bar{\Gamma}^{\prime}$, as desired.

The following key fact translates Galois genericity into a property of Hecke orbits.

Proposition 6.6. If $s$ is strictly Galois generic with respect to $K$, then for every generalized Hecke operator $T_{\varphi}$ on $S_{\Gamma}$ as in Definition 3.1, the set $T_{\varphi}(s)$ is permuted transitively by $\operatorname{Gal}(\bar{K} / K)$. 
Proof. By definition $s$ is strictly Galois generic with respect to $K$ if and only if $\operatorname{Gal}(\bar{K} / K)$ acts transitively on the fiber of [id] : $S_{\Gamma^{\prime}} \rightarrow S_{\Gamma}$ above $s$ for every congruence subgroup $\Gamma^{\prime} \subset \Gamma$. Applying this to $\Gamma^{\prime}=\Gamma \cap \varphi^{-1}(\Gamma)$, it follows that $\operatorname{Gal}(\bar{K} / K)$ also acts transitively on $T_{\varphi}(s):=[\varphi]\left([\mathrm{id}]^{-1}(s)\right)$, as desired.

In the rest of this section we discuss the relation between Hodge and Galois genericity.

Proposition 6.7. Every Galois generic point on $S_{\Gamma}$ is Hodge generic.

Proof. Consider any point $s=[x]_{\Gamma} \in \Gamma \backslash X^{+}=S_{\Gamma}$. Let $P^{\prime} \subset P$ be the smallest algebraic subgroup defined over $\mathbb{Q}$, such that $x: \mathbb{S}_{\mathbb{C}} \rightarrow P_{\mathbb{C}}$ factors through $P_{\mathbb{C}}^{\prime}$, i.e., the Mumford-Tate group of $x$. One easily verifies that there exists a unique connected mixed Shimura datum $\left(P^{\prime}, X^{\prime+}\right)$ with $x \in X^{\prime+}$. Thus with $\Gamma^{\prime}:=\Gamma \cap P^{\prime}(\mathbb{Q})^{+}$, the given point $s$ is the image of the point $s^{\prime}:=$ $[x]_{\Gamma^{\prime}} \in \Gamma^{\prime} \backslash X^{\prime+}=: S_{\Gamma^{\prime}}^{\prime}$ under the Shimura immersion induced by the inclusion $P^{\prime} \hookrightarrow P$. By Proposition 6.4 we may choose $E$ to contain the reflex fields of both $\left(P, X^{+}\right)$and $\left(P^{\prime}, X^{\prime+}\right)$. Then both $S_{\Gamma}$ and $S_{\Gamma^{\prime}}^{\prime}$ possess natural models over $E_{\mathrm{ab}}$, and the functoriality of canonical models [33, 11.10] implies that the morphism $S_{\Gamma^{\prime}}^{\prime} \rightarrow S_{\Gamma}$ is defined over $E_{\mathrm{ab}}$. Let $K \subset \mathbb{C}$ be any finitely generated extension of $E_{\mathrm{ab}}$ over which $s^{\prime}$ and hence $s$ is defined. Then $\bar{\Gamma}_{s, K} \subset P\left(\mathbb{A}_{f}\right)$ is the image of the corresponding subgroup $\bar{\Gamma}_{s^{\prime}, K} \subset P^{\prime}\left(\mathbb{A}_{f}\right)$. In particular, we have $\bar{\Gamma}_{s, K} \subset P^{\prime}\left(\mathbb{A}_{f}\right)$.

Suppose now that $s$ is Galois generic. By Proposition 6.5, after shrinking $\Gamma$ we may assume that $\bar{\Gamma}_{s, K}=\bar{\Gamma}$. The above inclusion then implies that $\bar{\Gamma} \subset$ $P^{\prime}\left(\mathbb{A}_{f}\right)$, and thus $\Gamma \subset P^{\prime}(\mathbb{Q})$. But the Axioms of Definition 2.1 imply that $\Gamma$ contains a Zariski dense subgroup of $P^{\text {der }}$. Therefore $P^{\text {der }} \subset P^{\prime}$. In view of the Axiom 2.1 (vi) this shows that $P^{\prime}=P$, and so $s$ is Hodge generic, as desired.

Conjecture 6.8. Every Hodge generic point on $S_{\Gamma}$ is Galois generic.

Only a few cases of this conjecture are known. We begin with a tautological case, which shows in particular that Galois generic points exist. The most interesting case concerning the Siegel moduli space, Theorem 6.13 , is due to Serre. Using Kummer theory we prove a relative version in Theorem 6.14.

Proposition 6.9. Let $s$ be any $\mathbb{C}$-valued point over the generic point of the model $S_{\Gamma}$ over $E_{\mathrm{ab}}$. Then $s$ is Galois generic.

Proof. Let $K$ denote the function field of $S_{\Gamma}$ as an algebraic variety over $E_{\mathrm{ab}}$. Then $s$ can be defined over $K$, and by construction we have $\bar{\Gamma}_{s, K}=\bar{\Gamma}$. Thus $s$ is strictly Galois generic with respect to $K$. 
Remark 6.10. Conjecture 6.8 is related to the Mumford-Tate conjecture, as follows. Suppose that $S_{\Gamma}$ is a Shimura subvariety of some Siegel moduli space and that $s \in S_{\Gamma}$ is a Hodge generic point that can be defined over a number field $L$. Then $P$ is the Mumford-Tate group of the abelian variety associated to $s$, and the Mumford-Tate conjecture says that the image of $\operatorname{Gal}(\bar{L} / L)$ in $P\left(\mathbb{Q}_{\ell}\right)$ is open for any rational prime $\ell$. Setting $K:=L E_{\mathrm{ab}}$, this statement implies that the image of $\bar{\Gamma}_{s, K} \subset P\left(\mathbb{A}_{f}\right)$ under the projection to $P\left(\mathbb{Q}_{\ell}\right)$ is an open subgroup of $P^{\text {der }}\left(\mathbb{Q}_{\ell}\right)$. An adèlic refinement of the Mumford-Tate conjecture would imply Conjecture 6.8 in this case.

In the following case this refinement was proved by Serre [42, Cor. de Th. 3], [41, §7, Cor. de Th. 3, Compl. 8.1]:

Theorem 6.11 (Serre). Let $A$ be an abelian variety over a finitely generated extension $L$ of $\mathbb{Q}$. Assume that $\operatorname{End}\left(A_{\bar{L}}\right)=\mathbb{Z}$ and that $\operatorname{dim} A$ is odd or equal to 2 or 6 . Then the continuous homomorphism

$$
\operatorname{Gal}(\bar{L} / L) \longrightarrow \operatorname{CSp}_{2 g}\left(\mathbb{A}_{f}\right)
$$

describing the Galois action on the adèlic Tate module of A has an open image.

Remark 6.12. The general case of Theorem 6.11 can be reduced to the number field case, as follows. Identify $A$ with the generic fiber of an abelian scheme $\mathcal{A}$ over an integral scheme $\mathcal{X}$ of finite type over $\mathbb{Q}$ with function field $L$. Then the above homomorphism factors through the étale fundamental group of $\mathcal{X}$, and for any point $x \in \mathcal{X}$ with residue field $L_{x}$ the homomorphism associated to the fiber $\mathcal{A}_{x}$ is the composite

$$
\operatorname{Gal}\left(\bar{L}_{x} / L_{x}\right) \longrightarrow \pi_{1, \text { ét }}(\mathcal{X}) \longrightarrow \operatorname{CSp}_{2 g}\left(\mathbb{A}_{f}\right) .
$$

In particular, the image of $\operatorname{Gal}\left(\bar{L}_{x} / L_{x}\right)$ is contained in the image of $\operatorname{Gal}(\bar{L} / L)$. By Hilbert irreducibility, as explained by Serre $[40, \S 1]$, one can choose a closed point $x \in X$ such that the two images in $\mathrm{CSp}_{2 g}\left(\mathbb{Q}_{\ell}\right)$ coincide for some prime $\ell$. The Tate conjecture for endomorphisms then implies that $\operatorname{End}\left(\mathcal{A}_{x, \bar{L}_{x}}\right)=\operatorname{End}\left(A_{\bar{L}}\right)=\mathbb{Z}$. As $L_{x}$ is a number field, Theorem 6.11 for $\mathcal{A}_{x}$ shows that the image of $\operatorname{Gal}\left(\bar{L}_{x} / L_{x}\right)$ is open in $\operatorname{CSp}_{2 g}\left(\mathbb{A}_{f}\right)$; hence the same follows for $\operatorname{Gal}(\bar{L} / L)$, as desired.

Theorem 6.13. Let $S_{\Gamma}$ be the connected Shimura variety associated to $\mathrm{CSp}_{2 g, \mathbb{Q}}$ and any $\Gamma$, as in Example 2.12. Assume that $g$ is odd or equal to 2 or 6 . Then any Hodge generic point on $S_{\Gamma}$ is Galois generic.

Proof. Let $A_{s}$ be the abelian variety associated to a Hodge generic point $s \in S_{\Gamma}$. Then $\operatorname{End}\left(A_{s, \mathbb{C}}\right)=\mathbb{Z}$. Let $L \subset \mathbb{C}$ be a finitely generated extension of $\mathbb{Q}$ over which $A_{s}$ is defined. Then by Theorem 6.11 the homomorphism $\operatorname{Gal}(\bar{L} / L) \rightarrow \operatorname{CSp}_{2 g}\left(\mathbb{A}_{f}\right)$ has an open image. Since the commutator subgroup of any open subgroup of $\mathrm{CSp}_{2 g}\left(\mathbb{A}_{f}\right)$ is an open subgroup of $\operatorname{Sp}_{2 g}\left(\mathbb{A}_{f}\right)$, with 
$K:=L E_{\text {ab }}$ it follows that the restriction $\operatorname{Gal}(\bar{K} / K) \rightarrow \operatorname{Sp}_{2 g}\left(\mathbb{A}_{f}\right)$ has an open image. This image is precisely the group $\bar{\Gamma}_{s, K}$. As any sufficiently small congruence subgroup $\Gamma$ of $\mathrm{CSp}_{2 g}(\mathbb{Q})$ is contained in $\mathrm{Sp}_{2 g}(\mathbb{Q})$, it follows that $\bar{\Gamma}_{s, K}$ is open in $\bar{\Gamma}$, as desired.

The following result establishes a relative version of Conjecture 6.8:

Theorem 6.14. Let $A \rightarrow S$ be as in Construction 2.9, and let $a \in A$ be $a$ Hodge generic point. Then its image $s \in S$ is Hodge generic, and if $s$ is Galois generic, then so is a.

Proof. The first assertion follows from the definition of Hodge genericity. For the second assume first that $A \rightarrow S$ and $a$ are all defined over a number field $L$. Then with the notation of Construction 2.9 the Galois action on the preimages of $a$ on coverings of $A$ corresponds to a continuous homomorphism

$$
\operatorname{Gal}(\bar{L} / L) \longrightarrow P\left(\mathbb{A}_{f}\right) \ltimes\left(V \otimes \mathbb{A}_{f}\right) .
$$

Let $\Delta$ denote its image. With $K:=L E_{\mathrm{ab}}$ we deduce that $\bar{\Gamma}_{a, K}$ is a normal subgroup of $\Delta$ containing the commutator subgroup of $\Delta$.

Since $a$ is Hodge generic, the subgroup $\mathbb{Z} a \subset A_{s}$ is Zariski dense. Thus a theorem of Ribet [38] in the form of Hindry [20, 2 2, Prop. 1] on the Kummer theory of $A_{s}$ implies that $\Delta \cap\left(V \otimes \mathbb{A}_{f}\right)$ is open in $V \otimes \mathbb{A}_{f}$. As in the proof of Proposition 6.4 we deduce that commutators of $\Delta$ with $\Delta \cap\left(V \otimes \mathbb{A}_{f}\right)$ again generate an open subgroup of $V \otimes \mathbb{A}_{f}$. Therefore $\bar{\Gamma}_{a, K} \cap\left(V \otimes \mathbb{A}_{f}\right)$ is open in $V \otimes \mathbb{A}_{f}$. On the other hand, the Galois genericity of $s$ means that the image $\bar{\Gamma}_{s, K}$ of $\bar{\Gamma}_{a, K}$ in $P\left(\mathbb{A}_{f}\right)$ is an open subgroup of $\bar{\Gamma} \subset P^{\operatorname{der}}\left(\mathbb{A}_{f}\right)$. Together it follows that $\bar{\Gamma}_{a, K}$ is an open subgroup of $\bar{\Gamma} \ltimes \bar{\Gamma}_{V} \subset P^{\operatorname{der}}\left(\mathbb{A}_{f}\right) \ltimes\left(V \otimes \mathbb{A}_{f}\right)$, and so $a$ is Galois generic, as desired.

The case where $a$ is defined over an arbitrary finitely generated extension of $\mathbb{Q}$ can be reduced to the number field case exactly as in Remark 6.12.

\section{Galois generic points and equidistribution}

In this section we deduce Conjecture 1.6 in certain cases for the Siegel moduli space from equidistribution results by Clozel, Oh, and Ullmo. As a preparation we fix some notation. Let $P:=\mathrm{CSp}_{2 g, \mathbb{Q}}$ for $g \geq 1$, let $Z \cong \mathbb{R}^{*}$ denote the center of $P(\mathbb{R})$, and set $Y:=P(\mathbb{R}) / Z$. As $Z$ is a normal subgroup of $P(\mathbb{R})$, there are natural left and right actions of $P(\mathbb{R})$ on $Y$. For any congruence subgroup $\Gamma \subset P(\mathbb{Q})$ and any element $p \in P(\mathbb{Q})$ we have a Hecke operator

$$
\begin{aligned}
& \Gamma \backslash Y \stackrel{[\mathrm{id}]}{\longleftarrow}\left(\Gamma \cap p^{-1} \Gamma p\right) \backslash Y \stackrel{[p \cdot]}{\longrightarrow} \Gamma \backslash Y, \\
& {[y]_{\Gamma} \longleftrightarrow[y]_{\Gamma \cap p^{-1} \Gamma p} \longleftrightarrow[p]_{\Gamma},}
\end{aligned}
$$


which maps an element $[y]_{\Gamma}$ to the finite set

$$
T_{p}\left([y]_{\Gamma}\right):=[p \cdot]\left([\mathrm{id}]^{-1}\left([y]_{\Gamma}\right)\right)=\left\{[p \gamma y]_{\Gamma} \mid \gamma \in\left(\Gamma \cap p^{-1} \Gamma p\right) \backslash \Gamma\right\} .
$$

This Hecke operator also acts on functions $f$ on $\Gamma \backslash Y$ by

$$
T_{p} f\left([y]_{\Gamma}\right):=\frac{1}{\operatorname{deg}_{\Gamma}(p)} \cdot \sum_{\gamma \in\left(\Gamma \cap p^{-1} \Gamma p\right) \backslash \Gamma} f\left([p \gamma y]_{\Gamma}\right),
$$

where

$$
\operatorname{deg}_{\Gamma}(p):=|\Gamma \backslash \Gamma p \Gamma|=\left[\Gamma: \Gamma \cap p^{-1} \Gamma p\right] .
$$

Clozel, Oh, and Ullmo [6, Thm. 1.6, Rem. (3)] have proved:

Theorem 7.1 (Clozel-Oh-Ullmo). In the above situation assume that $\Gamma=$ $P(\mathbb{Z})$. Let $\left(p_{n}\right)$ be a sequence in $P(\mathbb{Q})$ such that $\lim _{n \rightarrow \infty} \operatorname{deg}_{\Gamma}\left(p_{n}\right)=\infty$. Let $d \mu$ denote the right $P(\mathbb{R})$-invariant measure on $\Gamma \backslash Y$ with total volume 1 . Then for any element $[y]_{\Gamma} \in \Gamma \backslash Y$ the sets $T_{p_{n}}\left([y]_{\Gamma}\right)$ are equidistributed with respect to $d \mu$, in the sense that

$$
\lim _{n \rightarrow \infty} T_{p_{n}} f\left([y]_{\Gamma}\right)=\int_{\Gamma \backslash Y} f(\bar{y}) d \mu(\bar{y})
$$

for any continuous function $f$ on $Y$ with compact support.

Corollary 7.2. In Theorem 7.1 , the set $\bigcup_{n=1}^{\infty} T_{p_{n}}\left([y]_{\Gamma}\right)$ is dense in $\Gamma \backslash Y$.

Results like these should really be true for all $\Gamma$ and can presumably be proved along the lines of [6] and [7]. For our purposes a weaker consequence for all $\Gamma$ will suffice, which we can deduce from the above as follows.

Let $\left(P, X^{+}\right)$denote the connected Shimura datum with $P=\mathrm{CSp}_{2 g, \mathbb{Q}}$ from Example 2.12. Then $P(\mathbb{R})^{+}$is the identity component of $P(\mathbb{R})$, which has index 2 , and it contains the center $Z$. Thus $Y^{+}:=P(\mathbb{R})^{+} / Z$ is one of two connected components of $Y$. For any congruence subgroup $\Gamma \subset P(\mathbb{Q})^{+}:=$ $P(\mathbb{Q}) \cap P(\mathbb{R})^{+}$and any $p \in P(\mathbb{Q})^{+}$we can define a Hecke operator $T_{p}$,

$$
\Gamma \backslash Y^{+} \stackrel{[\mathrm{id}]}{\longleftarrow}\left(\Gamma \cap p^{-1} \Gamma p\right) \backslash Y^{+} \stackrel{[p \cdot]}{\longrightarrow} \Gamma \backslash Y^{+}
$$

in the same way as above. Fix a sequence $\left(p_{n}\right)$ in $P(\mathbb{Q})^{+}$. For any congruence subgroup $\Gamma \subset P(\mathbb{Q})^{+}$we set

$$
\Theta_{\Gamma}:=\bigcup_{n=1}^{\infty} T_{p_{n}}\left([1]_{\Gamma}\right) \subset \Gamma \backslash Y^{+}
$$

and let $\bar{\Theta}_{\Gamma}$ denote its closure in $\Gamma \backslash Y^{+}$. The following lemma concerns its dependence on $\Gamma$. 
Lemma 7.3. For any two congruence subgroups $\Gamma, \Gamma^{\prime} \subset P(\mathbb{Q})^{+}$,

(a) $\Theta_{\Gamma}$ is finite if and only if $\Theta_{\Gamma^{\prime}}$ is finite, and

(b) $\bar{\Theta}_{\Gamma}$ is a null set if and only if $\bar{\Theta}_{\Gamma^{\prime}}$ is a null set.

Proof. It suffices to prove this when $\Gamma^{\prime} \subset \Gamma$. Write $\Gamma=\bigcup_{i=1}^{r} \Gamma^{\prime} \gamma_{i}$ with representatives $\gamma_{i} \in \Gamma$, and let $\pi: \Gamma^{\prime} \backslash Y^{+} \rightarrow \Gamma \backslash Y^{+}$denote the natural projection. Then the calculation

$$
\begin{aligned}
T_{p_{n}}\left([1]_{\Gamma}\right) & =\Gamma \backslash \Gamma p_{n} \Gamma Z / Z=\bigcup_{i=1}^{r} \Gamma \backslash \Gamma p_{n} \Gamma^{\prime} \gamma_{i} Z / Z=\bigcup_{i=1}^{r} \pi\left(\Gamma^{\prime} \backslash \Gamma^{\prime} p_{n} \Gamma^{\prime} \gamma_{i} Z / Z\right) \\
& =\bigcup_{i=1}^{r} \pi\left(T_{p_{n}}\left(\left[\gamma_{i}\right]_{\Gamma^{\prime}}\right)\right)=\bigcup_{i=1}^{r} \pi\left(T_{p_{n}}\left([1]_{\Gamma^{\prime}}\right) \cdot \gamma_{i}\right)
\end{aligned}
$$

shows that $\Theta_{\Gamma}=\bigcup_{i=1}^{r} \pi\left(\Theta_{\Gamma^{\prime}} \cdot \gamma_{i}\right)$. Since $\pi$ is a finite (possibly ramified) covering, this implies (a). It also implies that $\bar{\Theta}_{\Gamma}=\bigcup_{i=1}^{r} \pi\left(\bar{\Theta}_{\Gamma^{\prime}} \cdot \gamma_{i}\right)$ and thus (b).

Using this we can deduce the following weak analogue of Corollary 7.2:

Proposition 7.4. If $\bar{\Theta}_{\Gamma}$ is a null set, then it is finite.

Proof. By Lemma 7.3 the assertion is independent of $\Gamma$. Thus it suffices to prove it for $\Gamma=P(\mathbb{Z})^{+}:=P(\mathbb{Z}) \cap P(\mathbb{R})^{+}=\operatorname{Sp}_{2 g}(\mathbb{Z})$. Here the natural map $P(\mathbb{Z})^{+} \backslash Y^{+} \rightarrow P(\mathbb{Z}) \backslash Y$ is bijective. Thus if $\lim _{n \rightarrow \infty} \operatorname{deg}_{\Gamma}\left(p_{n}\right)=\infty$, Corollary 7.2 shows that $\bar{\Theta}_{\Gamma}$ is not a null set. The same conclusion holds if $\limsup _{n \rightarrow \infty} \operatorname{deg}_{\Gamma}\left(p_{n}\right)=\infty$, because we may pass to a subsequence that diverges to $\infty$, which can only make $\bar{\Theta}_{\Gamma}$ smaller. If $\operatorname{deg}_{\Gamma}\left(p_{n}\right)$ is bounded, it is well-known that all $p_{n}$ lie in finitely many cosets of $\Gamma Z$. Then the $T_{p_{n}}$ form only finitely many distinct Hecke operators, and so $\Theta_{\Gamma}$ is finite. In all cases the desired implication holds.

Theorem 7.5. Let $S_{\Gamma}$ be the connected Shimura variety associated to $\mathrm{CSp}_{2 g, \mathbb{Q}}$ and any $\Gamma$, as in Example 2.12. Let $s \in S_{\Gamma}$ be a point and $\left(p_{n}\right)$ a sequence in $\mathrm{CSp}_{2 g}(\mathbb{Q})$. Then the subset $\bigcup_{n=1}^{\infty} T_{p_{n}}(s)$ of $S_{\Gamma}$ is either finite or Zariski dense.

Proof. Write $s=[x]_{\Gamma}$ for some $x \in X^{+}$, and consider the map

$$
\kappa: \Gamma \backslash Y^{+} \longrightarrow \Gamma \backslash X^{+}=S_{\Gamma},[y]_{\Gamma} \mapsto[y x]_{\Gamma} .
$$

By the definition of Hecke operators it satisfies $\kappa\left(T_{p_{n}}\left([1]_{\Gamma}\right)\right)=T_{p_{n}}\left(\kappa\left([1]_{\Gamma}\right)\right)=$ $T_{p_{n}}(s)$ and therefore $\kappa\left(\Theta_{\Gamma}\right)=\bigcup_{n=1}^{\infty} T_{p_{n}}(s)$. Consider a proper closed subvariety $Z \subset S_{\Gamma}$. If $\kappa\left(\Theta_{\Gamma}\right) \subset Z$, we have $\Theta_{\Gamma} \subset \kappa^{-1}(Z)$ and hence $\bar{\Theta}_{\Gamma} \subset \kappa^{-1}(Z)$. On the other hand, the irreducibility of $S_{\Gamma}$ implies that $Z$ and hence $\kappa^{-1}(Z)$ is a null set. Thus if $\kappa\left(\Theta_{\Gamma}\right)$ is not Zariski dense, it follows that $\bar{\Theta}_{\Gamma}$ is a null set. By Proposition 7.4 it is then finite, and so $\kappa\left(\Theta_{\Gamma}\right)$ is finite, as desired. 
Finally, we can settle Conjecture 1.6 in the following case.

Theorem 7.6. Let $S_{\Gamma}$ be the connected Shimura variety associated to $\mathrm{CSp}_{2 g, \mathbb{Q}}$ and any $\Gamma$, as in Example 2.12. Let $s \in S_{\Gamma}$ be a Galois generic point. Then any infinite subset of the generalized Hecke orbit of $s$ is Zariski dense in $S_{\Gamma}$. In particular, Conjecture 1.6 is true for $s \in S=S_{\Gamma}$ and any $Z$.

Proof. First note that every automorphism of the connected Shimura datum $\left(P, X^{+}\right)$here is an inner automorphism. Thus every generalized Hecke operator is a usual Hecke operator, and so the generalized Hecke orbit of $s$ coincides with the usual Hecke orbit. Consider any closed algebraic subvariety $Z \subset S_{\Gamma}$ containing an infinite subset of the Hecke orbit of $s$. We must prove that $Z=S_{\Gamma}$.

Let $E \subset E_{\mathrm{ab}} \subset \mathbb{C}$ be as in Section 6 , and choose a finitely generated extension $K_{0} \subset \mathbb{C}$ of $E_{\mathrm{ab}}$ such that $Z$ is defined over $K_{0}$. By Proposition 6.5 (a) $\Rightarrow$ (c) there exist a finitely generated extension $K \subset \mathbb{C}$ of $K_{0}$, a congruence subgroup $\Gamma^{\prime} \subset \Gamma$, and a point $s^{\prime} \in S_{\Gamma^{\prime}}$ mapping to $s$ under $\pi:=[\mathrm{id}]: S_{\Gamma^{\prime}} \rightarrow$ $S_{\Gamma}$, such that $s^{\prime}$ is strictly Galois generic with respect to $K$. The inverse image under $\pi$ of the Hecke orbit of $s$ is then simply the Hecke orbit of $s^{\prime}$. Thus $Z^{\prime}:=\pi^{-1}(Z)$ is a closed algebraic subvariety of $S_{\Gamma^{\prime}}$ defined over $K_{0}$ that contains an infinite subset of the Hecke orbit of $s^{\prime}$. It suffices to prove that $Z^{\prime}=S_{\Gamma^{\prime}}$. Thus after replacing $\Gamma, s, Z$ by $\Gamma^{\prime}, s^{\prime}, Z^{\prime}$ we may assume that $s$ is strictly Galois generic with respect to $K$. Then Proposition 6.6 says that for every Hecke operator $T_{p}$ on $S_{\Gamma}$, the set $T_{p}(s)$ is permuted transitively by $\operatorname{Gal}(\bar{K} / K)$.

Since $Z$ contains an infinite subset of the Hecke orbit of $s$, there exists a sequence $\left(p_{n}\right)$ in $P(\mathbb{Q})$ such that $Z$ contains a point from $T_{p_{n}}(s)$ for all $n$ and that all these points are distinct. As $Z$ is defined over $K_{0}$ and hence over $K$, the transitivity of $\operatorname{Gal}(\bar{K} / K)$ then implies that $Z$ contains the whole set $\bigcup_{n=1}^{\infty} T_{p_{n}}(s)$. By construction this set is infinite; hence by Theorem 7.5 it is Zariski dense in $S_{\Gamma}$. Therefore $Z=S_{\Gamma}$, as desired.

Finally, in the situation of Conjecture 1.6, this shows that either $Z=S_{\Gamma}$, or $Z$ is finite and hence a point. In the former case it is special by tautology, in the latter it is weakly special by Remark 4.11.

\section{Remarks 7.7.}

(a) Proposition 6.9 and Theorem 6.13 show that Theorem 7.6 is not vacuous.

(b) In Theorem 7.5 one should expect that the subset $\bigcup_{n=1}^{\infty} T_{p_{n}}(s)$ of $S_{\Gamma}$ is either finite or dense for the analytic topology. In fact, in the case $\Gamma=P(\mathbb{Z})^{+}$this is a consequence of Theorem 7.1.

(c) Clozel, Oh, and Ullmo [6, Thm. 1.6] prove an analogue of Theorem 7.1 for most connected almost simple simply connected semisimple groups over $\mathbb{Q}$ with arbitrary congruence subgroups. A version for all adjoint groups 
would apply to more Hecke operators and hence quickly yield generalizations of Theorems 7.5 and 7.6 to arbitrary connected pure Shimura varieties. Such a version can presumably be proved along the lines of $[6]$ and [7].

(d) Clozel has pointed out to the author that the desired extension to most adjoint groups might also be deduced from [6, Cor. 1.4], using the fact that for every Hecke operator induced by an element of the adjoint group, some positive power is a linear combination of Hecke operators induced by elements of the simply connected covering.

(e) An overview of various other aspects of equidistribution is given in Ullmo [45].

\section{References}

1. Y. AndRÉ - G-functions and geometry, Aspects of Mathematics, E13, Friedr. Vieweg \& Sohn, Braunschweig, 1989.

2. — , "Finitude des couples d'invariants modulaires singuliers sur une courbe algébrique plane non modulaire", J. Reine Angew. Math. 505 (1998), p. 203208.

3. - , "Shimura varieties, subvarieties, and CM points", Six lectures at the University of Hsinchu (Taiwan), August-September 2001 (with an appendix by C.-L. Chai), http://www.math.umd.edu/ yu/notes.shtml.

4. F. A. Bogomolov - "Points of finite order on abelian varieties", Izv. Akad. Nauk SSSR Ser. Mat. 44 (1980), no. 4, p. 782-804.

5. E. Bombieri - "The Mordell conjecture revisited", Ann. Scuola Norm. Sup. Pisa Cl. Sci. (4) 17 (1990), no. 4, p. 615-640.

6. L. Clozel, H. OH and E. Ullmo - "Hecke operators and equidistribution of Hecke points", Invent. Math. 144 (2001), no. 2, p. 327-351.

7. L. Clozel and E. Ullmo - "Équidistribution des points de Hecke", Contributions to automorphic forms, geometry, and number theory, Johns Hopkins Univ. Press, Baltimore, MD, 2004, p. 193-254.

8. R. F. Coleman - "Ramified torsion points on curves", Duke Math. J. 54 (1987), no. 2, p. 615-640.

9. P. Deligne - "Travaux de Shimura", Séminaire Bourbaki, 23ème année (1970/71), Exp. No. 389, Springer, Berlin, 1971, p. 123-165. Lecture Notes in Math., Vol. 244.

10. — , "Variétés de Shimura: interprétation modulaire, et techniques de construction de modèles canoniques", Automorphic forms, representations and $L$ functions (Proc. Sympos. Pure Math., Oregon State Univ., Corvallis, Ore., 1977), Part 2, Proc. Sympos. Pure Math., XXXIII, Amer. Math. Soc., Providence, R.I., 1979, p. 247-289.

11. P. Deligne, J. S. Milne, A. Ogus and K.-Y. ShiH - Hodge cycles, motives, and Shimura varieties, Lecture Notes in Mathematics, vol. 900, Springer-Verlag, Berlin, 1982.

12. B. Edixhoven and J.-H. Evertse (eds.) - Diophantine approximation and abelian varieties, Lecture Notes in Mathematics, vol. 1566, Springer-Verlag, 
Berlin, 1993, Introductory lectures, Papers from the conference held in Soesterberg, April 12-16, 1992.

13. B. EdIXHOVEn - "Special points on the product of two modular curves", Compositio Math. 114 (1998), no. 3, p. 315-328.

14. — , "On the André-Oort conjecture for Hilbert modular surfaces", Moduli of abelian varieties (Texel Island, 1999), Progr. Math., vol. 195, Birkhäuser, Basel, 2001, p. 133-155.

15. B. Edixhoven and A. YAfaev - "Subvarieties of Shimura varieties", Ann. of Math. (2) 157 (2003), no. 2, p. 621-645.

16. G. FAltings - "Endlichkeitssätze für abelsche Varietäten über Zahlkörpern", Invent. Math. 73 (1983), no. 3, p. 349-366, Erratum: 75 no. 2 (1984).

17. — , "Finiteness theorems for abelian varieties over number fields", Arithmetic geometry (Storrs, Conn., 1984) (G. Cornell and J. H. Silverman, eds.), Springer, New York, 1986, p. 9-27.

18. — , "Diophantine approximation on abelian varieties", Ann. of Math. (2) 133 (1991), no. 3, p. 549-576.

19. - - "The general case of S. Lang's conjecture", Barsotti Symposium in Algebraic Geometry (Abano Terme, 1991), Perspect. Math., vol. 15, Academic Press, San Diego, CA, 1994, p. 175-182.

20. M. Hindry - "Autour d'une conjecture de Serge Lang", Invent. Math. 94 (1988), no. 3, p. 575-603.

21. - , "Sur les conjectures de Mordell et Lang (d'après Vojta, Faltings et Bombieri)", Astérisque (1992), no. 209, p. 11, 39-56, Journées Arithmétiques, 1991 (Geneva).

22. M. Hindry and J. H. Silverman - Diophantine geometry, Graduate Texts in Mathematics, vol. 201, Springer-Verlag, New York, 2000.

23. E. Hrushovski - "The Manin-Mumford conjecture and the model theory of difference fields", Ann. Pure Appl. Logic 112 (2001), no. 1, p. 43-115.

24. S. LANG - "Division points on curves", Ann. Mat. Pura Appl. (4) 70 (1965), p. 229-234.

25. - - Fundamentals of Diophantine geometry, Springer-Verlag, New York, 1983.

26. M. MCQuillan - "Division points on semi-abelian varieties", Invent. Math. 120 (1995), no. 1, p. 143-159.

27. J. S. MiLne - "The action of an automorphism of $\mathbf{C}$ on a Shimura variety and its special points", Arithmetic and geometry, Vol. I, Progr. Math., vol. 35, Birkhäuser Boston, Boston, MA, 1983, p. 239-265.

28. - , "Canonical models of (mixed) Shimura varieties and automorphic vector bundles", Automorphic forms, Shimura varieties, and $L$-functions, Vol. I (Ann Arbor, MI, 1988), Perspect. Math., vol. 10, Academic Press, Boston, MA, 1990, p. 283-414.

29. B. Moonen - "Special points and linearity properties of Shimura varieties", Thesis, University of Utrecht, 1995.

30. - , "Models of Shimura varieties in mixed characteristics", Galois representations in arithmetic algebraic geometry (Durham, 1996), London Math. Soc. Lecture Note Ser., vol. 254, Cambridge Univ. Press, Cambridge, 1998, p. 267 350.

31. L. Mordell - "On the rational solutions of the indeterminate equations of the third and fourth degrees", Proc. Camb. Philos. Soc. 21 (1922), p. 179-192. 
32. F. OorT - "Canonical liftings and dense sets of CM-points", Arithmetic geometry (Cortona, 1994), Sympos. Math., XXXVII, Cambridge Univ. Press, Cambridge, 1997, p. 228-234.

33. R. PINK - "Arithmetical compactification of mixed shimura varieties", Thesis, Rheinische Friedrich-Wilhelms-Universität Bonn, 1989, Bonner Mathematische Schriften, 209 (1990).

34. R. Pink and D. Roessler - "On Hrushovski's proof of the Manin-Mumford conjecture", Proceedings of the International Congress of Mathematicians, Vol. I (Beijing, 2002) (Beijing), Higher Ed. Press, 2002, p. 539-546.

35. M. RAYNAUD - "Around the Mordell conjecture for function fields and a conjecture of Serge Lang", Algebraic geometry (Tokyo/Kyoto, 1982), Lecture Notes in Math., vol. 1016, Springer, Berlin, 1983, p. 1-19.

36. - - "Courbes sur une variété abélienne et points de torsion", Invent. Math. 71 (1983), no. 1, p. 207-233.

37. - , "Sous-variétés d'une variété abélienne et points de torsion", Arithmetic and geometry, Vol. I, Progr. Math., vol. 35, Birkhäuser Boston, Boston, MA, 1983, p. 327-352.

38. K. A. Ribet - "Kummer theory on extensions of abelian varieties by tori", Duke Math. J. 46 (1979), no. 4, p. 745-761.

39. D. Roessler - "A note on the Manin-Mumford conjecture", 2004, preprint.

40. J.-P. SERRE - "Lettre à Ken Ribet du 1/1/1981 et du 29/1/1981", Oeuvres vol. IV, p. 1-20, Springer, 2000.

41. — , "Lettre à Marie-France Vignéras du 10/2/1986", Oeuvres vol. IV, p. 38-55, Springer, 2000.

42. — , "Résumé des cours de 1985-1986", Oeuvres vol. IV, p. 33-37, Springer, 2000.

43. G. Shimura and Y. TAniyama - Complex multiplication of abelian varieties and its applications to number theory, Publications of the Mathematical Society of Japan, vol. 6, The Mathematical Society of Japan, Tokyo, 1961.

44. E. Ullmo - "Positivité et discrétion des points algébriques des courbes", Ann. of Math. (2) 147 (1998), no. 1, p. 167-179.

45. - , "Théorie ergodique et géométrie arithmétique", Proceedings of the International Congress of Mathematicians, Vol. II (Beijing, 2002) (Beijing), Higher Ed. Press, 2002, p. 197-206.

46. P. Vojta - "Siegel's theorem in the compact case", Ann. of Math. (2) 133 (1991), no. 3, p. 509-548.

47. - , "Applications of arithmetic algebraic geometry to Diophantine approximations", Arithmetic algebraic geometry (Trento, 1991), Lecture Notes in Math., vol. 1553, Springer, Berlin, 1993, p. 164-208.

48. A. WEIL - "L'arithmétique sur les courbes algébriques", Acta Math. 52 (1928), p. $281-315$.

49. G. Wüstholz - "New developments in diophantine and arithmetic algebraic geometry", Aspects of Mathematics, E6, p. x+311, Friedr. Vieweg \& Sohn, Braunschweig, third ed., 1992, Appendix to "Papers from the seminar held at the Max-Planck-Institut für Mathematik", Bonn/Wuppertal, 1983/1984.

50. A. YAfAEV - "On a result of Ben Moonen on the moduli space of principally polarised abelian varieties", to appear in Compositio Math.

51. - , "A conjecture of Yves André", 2003, math.NT/0302125.

52. S.-W. ZHANG - "Equidistribution of small points on abelian varieties", Ann. of Math. (2) 147 (1998), no. 1, p. 159-165. 